\title{
Alterations in Concentration/Activity of Superoxide Dismutases in Context of Obesity and Selected Single Nucleotide Polymorphisms in Genes: SOD1, SOD2, SOD3
}

\author{
Lukasz Lewandowski *D, Marta Kepinska and Halina Milnerowicz \\ Department of Biomedical and Environmental Analyses, Faculty of Pharmacy, Wroclaw Medical University, \\ Borowska Street 211, 50-556 Wrocław, Poland; \\ marta.kepinska@umed.wroc.pl (M.K.); halina.milnerowicz@umed.wroc.pl (H.M.) \\ * Correspondence: lukasz.lewandowski@umed.wroc.pl
}

Received: 6 July 2020; Accepted: 15 July 2020; Published: 17 July 2020

\begin{abstract}
Little is known about the contribution of each of the three superoxide dismutase isozymes (SODs) to the total SOD activity in extracellular fluids. This study was aimed to investigate the alterations in concentration/activity of (SODs) in plasma, in context of sex, obesity, exposition to cigarette smoke, and genotypic variability of five selected single nucleotide polymorphisms (SNPs) in genes SOD1, SOD2, SOD3. Men showed higher SOD1 concentration, lower SOD3 concentration and higher total antioxidative capacity (TAC) values. Intersexual variability was observed in concentration of copper, zinc, and cadmium. The obese showed higher total oxidative capacity regardless of sex. An increase in SOD2 activity was coexistent with obesity in men, and exposition to cigarette smoke in non-obese individuals. Additionally, in state of this exposition, $\mathrm{Cu}, \mathrm{Zn}-\mathrm{SOD}$ contribution to the total SOD was lower. Interestingly, over $90 \%$ of the obese were of $\mathrm{C} / \mathrm{T}$ genotype of rs 4880 (SOD2). Non-obese of T/T genotype (rs4880) were of lower total SOD activity due to decrease in both $\mathrm{Cu}, \mathrm{Zn}-\mathrm{SOD}$ and $\mathrm{Mn}-\mathrm{SOD}$ activities. SNP rs2234694 was associated with differences in concentration of SODs, depending on obesity status. Correlations indicate that both TAC and SODs, together, may adapt to insulin resistance and inflammation-derived oxidative stress found in obesity. This topic should be further investigated.
\end{abstract}

Keywords: metals; obesity; oxidative stress; single nucleotide polymorphisms; superoxide dismutase isozymes

\section{Introduction}

The demand to thoroughly understand the risk factors for developing obesity stems from the worldwide increase in prevalence of this disease [1], especially in the recent years. It is commonly claimed that oxidative stress, coexisting with dyslipidemia, aberrant immunological response, disorders of the electrolyte balance, mitochondrial dysfunction, increased secretion of proinflammatory adipokines [2-5], and activation of inflammasomes and toll-like receptors contribute to the state of meta-inflammation, thus disorders such as insulin resistance, type 2 diabetes, and atherosclerosis often co-existent with obesity [6].

Contemporary medicine links oxidative stress with insulin resistance [7-9]. In a state of normoglycemia, glucose is metabolized mainly in two pathways: glycolysis and pentose phosphate pathway and reactive oxygen species (ROS) are sufficiently neutralized by the antioxidative system, comprised of low-molecular weight compounds (e.g., glutathione, bilirubin, uric acid, ascorbic acid, D- $\alpha$-tocopherols) and high-molecular-weight compounds (enzymatic antioxidants). However, in 
the state of hyperglycemia, mitochondrial dysfunction leads to overproduction of electron donors (mainly NADH+H+). Excessive ROS generation and deficiency in antioxidative capacity caused by inactivation of enzymatic antioxidants, partly in the process of enzyme glycation, lead to disorders in lipid and carbohydrate metabolism and oxidative damage in proteins and DNA [10,11]. Moreover, superoxide anion, through oxidative modification, decreases the activity of glyceraldehyde 3-phosphate dehydrogenase (GAPDH) [12], which leads to intensification of carbohydrate metabolism via alternative pathways: polyol (sorbitol) and hexosamine [13,14]. Activation of protein kinase $C$ by diacylglycerol and advanced glycation end products formed from glyceraldehyde 3-phosphate (in the state of GAPDH activity deficiency) causes phosphorylation (inactivation) of insulin receptor substrate-1 (IRS-1), therefore leading to insulin resistance [15-17]. Moreover, the state of insulin resistance in obesity is promoted by disorders in modulation of the processes of secretion and degradation of insulin (due to hypozincemia) [18,19], glycation of insulin [20], "leptin resistance" causing accumulation of triglycerides in muscle tissue [21-23] and disorders in secretion of neuropeptides [24].

This study focuses on superoxide dismutases (SODs), a vital family of enzymatic antioxidants, which neutralize superoxide anion. In humans SOD is found in form of three isozymes: cytosolic SOD1 (gene SOD1, chromosome 21q22.11), mitochondrial SOD2 (gene SOD2, chromosome 6q25.3), and extracellular SOD3 (gene SOD3, chromosome 4p15.2) [25,26]. SOD1 and SOD3 are copper-zinc SODs, whereas SOD2 is a manganese SOD. Interestingly, despite the fact that two of three mentioned SODs are ascribed to the intracellular compartment, it is known that all of them could be found in the extracellular compartment (serum, plasma), which is assumed to be due to migration of intracellular isozymes SOD1 and SOD2 to the extracellular matrix [27-29]. The association of SOD with obesity is due to its protective role as an antioxidant [30]. The extracellular SOD, SOD3, due to its abundance on the surface of endothelium, neutralizes the superoxide anion in the extracellular matrix, disrupting the generation of peroxynitrite, one of the mediators of pancreatic $\beta$-cell damage [31]. SOD2, found mainly in the mitochondria, by neutralizing superoxide anion, prevents from increased activation of uncoupling proteins (UCPs) by superoxide anion (which is observed in state of chronic hyperglycemia, as found in obesity). As UCPs affect the process of insulin secretion in response to actual glycemia, maintaining proper SOD2 activity is important in context of obesity [32-34]. Moreover, SODs protect from the development of diabetes since it was shown that increased expression of SOD1 in pancreatic islet $\beta$ cells is associated with increased resistance to alloxan-induced diabetes [35] and increased SOD activity leads to increased resistance to streptozocin-induced diabetes [36-39].

The variability in SOD concentration/activity between individuals may be associated with single nucleotide polymorphisms (SNPs) in genes coding for SOD isozymes. Flekac et al. [40] showed that diabetic individuals of C/C genotype (SNP rs2234694, SOD1 gene) were characterized of lower total SOD activity. Another SNP, rs1799895 (SOD3), is associated with the loss of SOD3 affinity to heparan sulfate (due to Arg213Gly substitution in the extracellular matrix binding region of SOD3), causing an increased concentration of SOD3 in extracellular fluid [41-43]. Individuals of no allele associated with Ile58Thr substitution in SOD3 were characterized of lower SOD activity [44]. One of the most-studied SNPs of the SOD2 gene, rs4880, associated with Val16Ala substitution and thus conformational changes in the mitochondrial targeting sequence of SOD2 is not only associated with variability of SOD activity in mitochondrial matrix [45], but also variability in total SOD activity and malondialdehyde, leptin and total cholesterol concentration in blood, in both obese and/or non-obese [40,46]. Moreover, it has been shown that genotypic variability of rs 4880 may be associated with difference in chances of developing obesity, as the individuals of Val/Val genotype had two-fold increased chance of developing obesity, compared to individuals of Ala/Ala or Ala/Val genotype [47].

This work is the result of reflections on the subject of the influence of a hereditary factor-DNA polymorphism in genes SOD1, SOD2, SOD3 on the variability of concentration/activity of SOD isozymes in plasma. The additional factors taken into consideration in this study are sex, obesity, and exposition to cigarette smoke. To our knowledge, apart from two SNPs, rs2234694 (SOD1) and rs4880 (SOD2), the SNPs investigated in this study (rs5746105, rs927450, rs8192287) have not previously 
been studied in the context of obesity. Interestingly, regarding extracellular fluid (serum, plasma), the literature covers mostly the topic of changes in total SOD activity. Only a few studies provide information on the activity of individual fractions Cu,Zn-SOD (SOD1 and SOD3) and Mn-SOD (SOD2), which results from the specific nomenclature, suggesting that SOD1 and SOD2 are mainly intracellular enzymes. Determining only the total SOD activity prevents from deeper understanding of how individual SOD isozymes interact to maintain proper antioxidative capacity in the extracellular fluid and if obesity affects this interaction. Moreover, the literature does not thoroughly cover the topic of the association between the concentration of copper and zinc with the alterations in concentration/activity of copper-zinc isozymes SOD1 and SOD3. The aim of this work is to attempt to cover all of these topics in one study. The changes in SOD isozyme concentration/activity are also analyzed in reference to total antioxidative capacity - mainly associated with low-molecular-weight antioxidants, and concentration of malondialdehyde (MDA) — a marker of lipid peroxidation process.

\section{Results}

2.1. Intersexual Variability in Concentration/Activity of SOD Isozymes, Total Antioxidative Capacity (TAC) Values, Concentration of Malondialdehyde (MDA) and Metals: Copper, Zinc, and Cadmium

The analysis was performed on values observed in individuals not exposed to cigarette smoke. Descriptive statistics is shown in Table 1. Although no significant differences between women and men were found in values of SOD activity, men were characterized of significantly higher SOD1 concentration $(p<0.00001)$ and lower SOD3 concentration $(p \approx 0.0214$ and $p \approx 0.0643$ for SOD3 $(\mathrm{ng} / \mathrm{mL})$ and SOD3 (ng/mg total protein), respectively) in plasma. Interestingly, higher total antioxidative capacity (TAC) values $(p<0.000001)$ and MDA concentration $(p \approx 0.0085)$ were observed in men, compared to women.

Table 1. Values of selected pro- and antioxidative parameters, and concentration of selected metals, in context of intersexual variability, in individuals not exposed to cigarette smoke.

\begin{tabular}{|c|c|c|c|}
\hline Parameter & Women $(n=33)$ & $\operatorname{Men}(n=35)$ & $p$ \\
\hline ** SOD1 (ng/mL) & $\{18.14 ; 23.43 ; 33.01\}$ & $\{35.28 ; 43.27 ; 51.97\}$ & $<0.000001$ \\
\hline ** SOD1 (ng/mg total protein) & $\{0.135 ; 0.187 ; 0.271\}$ & $\{0.270 ; 0.348 ; 0.442\}$ & $<0.000001$ \\
\hline $\mathrm{SOD} 2(\mathrm{ng} / \mathrm{mL})$ & $\{21.80 ; 23.61 ; 32.70\}$ & $\{20.19 ; 26.04 ; 32.85\}$ & 0.9251 \\
\hline SOD2 (ng/mg total protein) & $\{0.168 ; 0.203 ; 0.262\}$ & $\{0.152 ; 0.217 ; 0.298\}$ & 0.9052 \\
\hline ** SOD3 (ng/mL) & $\{27.71 ; 31.69 ; 37.88\}$ & $\{23.24 ; 27.85 ; 32.01\}$ & 0.0214 \\
\hline SOD3 (ng/mg total protein) & $\{0.195 ; 0.270 ; 0.322\}$ & $\{0.183 ; 0.239 ; 0.277\}$ & 0.0643 \\
\hline $\mathrm{SOD}(\mathrm{U} / \mathrm{L})$ & $\{1700 ; 1971 ; 2359\}$ & $\{1749 ; 2301 ; 2522\}$ & 0.2412 \\
\hline SOD (U/g total protein) & $\{13.20 ; 16.54 ; 20.24\}$ & $\{12.64 ; 18.89 ; 21.29\}$ & 0.3026 \\
\hline SOD (U/mg SODs) & $\{18.60 ; 23.30 ; 28.20\}$ & $\{18.03 ; 22.81 ; 25.90\}$ & 0.3374 \\
\hline $\mathrm{Cu}, \mathrm{Zn}-\mathrm{SOD}(\mathrm{U} / \mathrm{L})$ & $\{514 ; 771 ; 1106\}$ & $\{573 ; 854 ; 1184\}$ & 0.5977 \\
\hline $\mathrm{Cu}, \mathrm{Zn}-\mathrm{SOD}$ (U/g total protein) & $\{4.23 ; 6.07 ; 8.64\}$ & $\{4.35 ; 6.71 ; 10.23\}$ & 0.5234 \\
\hline $\mathrm{Cu}, \mathrm{Zn}-\mathrm{SOD}(\mathrm{U} / \mathrm{mg} \mathrm{SOD} 1+\mathrm{SOD} 3)$ & $\{8.91 ; 14.29 ; 19.03\}$ & $\{7.76 ; 12.27 ; 15.86\}$ & 0.2030 \\
\hline Cu,Zn-SOD (\% SOD activity) & $\{28.60 ; 40.32 ; 55.59\}$ & $\{32.40 ; 37.89 ; 49.68\}$ & 0.5476 \\
\hline Mn-SOD (U/L) & $\{979 ; \mathbf{1 2 2 6} ; 1516\}$ & $\{1098 ; 1240 ; 1582\}$ & 0.5194 \\
\hline Mn-SOD (U/g total protein) & $\{6.86 ; 9.99 ; 12.33\}$ & $\{8.60 ; 10.30 ; 12.53\}$ & 0.5314 \\
\hline Mn-SOD (U/mg SOD2) & $\{33.27 ; 44.54 ; 69.62\}$ & $\{37.76 ; 50.76 ; 66.51\}$ & 0.5265 \\
\hline ** TAC (mM UAE) & $\{0.219 ; \mathbf{0 . 2 6 8} ; 0.308\}$ & $\{0.345 ; \mathbf{0 . 3 8 2} ; 0.408\}$ & $<0.000001$ \\
\hline${ }^{* *} \operatorname{MDA}(\mu \mathrm{mol} / \mathrm{L})$ & $\{3.36 ; 4.43 ; 6.60\}$ & $\{5.29 ; 6.22 ; 7.30\}$ & 0.0085 \\
\hline$* * \mathrm{Cu}(\mu \mathrm{g} / \mathrm{L})$ & $\{976 ; 1108 ; 1303\}$ & $\{912 ; 972 ; 1059\}$ & 0.0144 \\
\hline$* * \operatorname{Zn}(\mu \mathrm{g} / \mathrm{L})$ & $\{845 ; 895 ; 932\}$ & $\{961 ; 1034 ; 1116\}$ & $<0.00001$ \\
\hline$* * \mathrm{Zn} / \mathrm{Cu}$ & $\{0.74 ; 0.87 ; 0.91\}$ & $\{0.94 ; 1.03 ; 1.18\}$ & $<0.00001$ \\
\hline ** Cd (mg/g Hb) & $\{1.68 ; 2.46 ; 3.56\}$ & $\{1.12 ; 1.62 ; 2.63\}$ & 0.0125 \\
\hline
\end{tabular}

Values shown as: 1 st quartile; median value; 3 rd quartile. ${ }^{* *}$ significant difference (median values). 
Significant intersexual variability was also found in values of concentration of copper, zinc, in serum, and cadmium, in full blood. Men were characterized of lower cadmium concentration $(p \approx 0.0125)$ and lower zinc-to-copper ratio $(p<0.00001)$ due to significantly lower copper concentration $(p \approx 0.0144)$ and higher zinc concentration $(p<0.00001)$.

\subsection{Alterations in Concentration/Activity of Superoxide Dismutase (SOD) Isozymes, TAC Values, Concentration of MDA and Metals: Copper, Zinc, and Cadmium, in Context of Obesity and Exposition to Cigarette Smoke}

The analysis in context of obesity was performed on data of individuals not exposed to cigarette smoke. Different observations were made depending on sex. In women (Table 2), no difference was found in concentration/activity of SODs, although the mean specific $\mathrm{Cu}, \mathrm{Zn}-\mathrm{SOD}$ activity (U/mg SOD1+SOD3) was markedly (approximately, $34 \%$ ) lower $(p \approx 0.0814)$ in obese individuals. Moreover, higher values of TAC $(p \approx 0.0222)$ and MDA $(p<0.0001)$ concentration were observed in obese women, compared to the non-obese. Lower zinc-to-copper ratio and higher concentration of copper (in serum) and cadmium (in full blood) were found in obese women, compared to the non-obese.

Table 2. Values of selected pro- and antioxidative parameters, and concentration of selected metals, in context of obesity, in women not exposed to cigarette smoke.

\begin{tabular}{|c|c|c|c|}
\hline Parameter & Control Group $(n=24)$ & Obese Group $(n=9)$ & $p$ \\
\hline SOD1 (ng/mL) & $23.45 \pm 10.52$ & $27.54 \pm 7.94$ & 0.3002 \\
\hline SOD1 (ng/mg total protein) & $\mathbf{0 . 1 8 4} \pm 0.083$ & $0.236 \pm 0.059$ & 0.0982 \\
\hline SOD2 $(\mathrm{ng} / \mathrm{mL})$ & $\{21.16 ; 23.21 ; 31.84\}$ & $\{22.63 ; 25.35 ; 32.70\}$ & 0.4141 \\
\hline SOD2 (ng/mg total protein) & $\mathbf{0 . 2 0 3} \pm 0.058$ & $\mathbf{0 . 2 5 1} \pm 0.080$ & 0.0691 \\
\hline SOD3 (ng/mL) & $31.25 \pm 8.92$ & $31.78 \pm 4.75$ & 0.8747 \\
\hline SOD3 (ng/mg total protein) & $0.247 \pm 0.076^{\mathrm{C}}$ & $0.302 \pm 0.054$ & 0.0903 \\
\hline $\mathrm{SOD}(\mathrm{U} / \mathrm{L})$ & $2091 \pm 677$ & $2018 \pm 267$ & 0.6667 \\
\hline SOD (U/g total protein) & $16.17 \pm 5.91$ & $16.62 \pm 3.29$ & 0.8308 \\
\hline SOD (U/mg SODs) & $25.52 \pm 9.57$ & $21.03 \pm 4.74$ & 0.1923 \\
\hline $\mathrm{Cu}, \mathrm{Zn}-\mathrm{SOD}(\mathrm{U} / \mathrm{L})$ & $896 \pm 516$ & $720 \pm 160$ & 0.1585 \\
\hline $\mathrm{Cu}, \mathrm{Zn}-\mathrm{SOD}$ (U/g total protein) & $6.43 \pm 3.80$ & $6.38 \pm 0.91$ & 0.9566 \\
\hline $\mathrm{Cu}, \mathrm{Zn}-\mathrm{SOD}(\mathrm{U} / \mathrm{mg} \mathrm{SOD} 1+\mathrm{SOD} 3)$ & $15.44 \pm 7.99$ & $\mathbf{1 1 . 5 4} \pm 4.10$ & 0.0814 \\
\hline $\mathrm{Cu}, \mathrm{Zn}-\mathrm{SOD}(\% \mathrm{SOD}$ activity) & $43.36 \pm 20.30$ & $36.91 \pm 8.22$ & 0.2033 \\
\hline $\mathrm{Mn}-\mathrm{SOD}(\mathrm{U} / \mathrm{L})$ & $1243 \pm 558$ & $1201 \pm 261$ & 0.8311 \\
\hline Mn-SOD (U/g total protein) & $9.85 \pm 4.58$ & $\mathbf{1 0 . 5 8} \pm 2.88$ & 0.6625 \\
\hline Mn-SOD (U/mg SOD2) & $51.04 \pm 28.29$ & $46.48 \pm 19.86$ & 0.6614 \\
\hline * TAC (mM UAE) & $0.252 \pm 0.047$ & $0.353 \pm 0.087$ & 0.0222 \\
\hline${ }^{*} \operatorname{MDA}(\mu \mathrm{mol} / \mathrm{L})$ & $4.01 \pm 1.62$ & $7.33 \pm 1.92$ & $<0.0001$ \\
\hline${ }^{*} \mathrm{Cu}(\mu \mathrm{g} / \mathrm{L})$ & $1032 \pm 174$ & $1229 \pm 91$ & 0.0087 \\
\hline $\mathrm{Zn}(\mu \mathrm{g} / \mathrm{L})$ & $904 \pm 78$ & $879 \pm 130$ & 0.5005 \\
\hline$* \mathrm{Zn} / \mathrm{Cu}$ & $0.893 \pm 0.106$ & $0.758 \pm 0.150$ & 0.0140 \\
\hline * Cd (mg/g Hb) & $2.19 \pm 0.92$ & $3.29 \pm 1.48$ & 0.0250 \\
\hline
\end{tabular}

Values shown as: mean value \pm standard deviation and $\{1$ st quartile; median value; 3 rd quartile $\} .{ }^{*}$ significant difference (mean values).

In men (Table 3), similarly to women, no difference was found in concentration/activity of SODs, but in the obese, the mean concentration of SOD1 $(\mathrm{ng} / \mathrm{mL})$ was approximately $22 \%$ higher $(p \approx 0.0575)$, the mean concentration of SOD2 $(\mathrm{ng} / \mathrm{mL})$ was approximately $34 \%$ lower $(p \approx 0.0697)$, and the mean $\mathrm{Cu}, \mathrm{Zn}-\mathrm{SOD}$ specific activity (U/mg SOD1+SOD3) was approximately $30 \%$ lower $(p \approx 0.0648)$, compared to the non-obese. Interestingly, both the activity and specific activity of Mn-SOD were higher in obese men; the $p$ values were approximately $0.0015,0.0006,0.0145$, for variables Mn-SOD (U/L), Mn-SOD (U/g total protein), Mn-SOD (U/mg SOD2), respectively. The TAC values in the obese were higher, although insignificantly $(p \approx 0.0581)$. No significant difference was found in concentration values of MDA, copper, zinc, and cadmium. 
Table 3. Values of selected pro- and antioxidative parameters, and concentration of selected metals, in context of obesity, in men not exposed to cigarette smoke.

\begin{tabular}{|c|c|c|c|}
\hline Parameter & Control Group $(n=17)$ & Obese Group $(n=18)$ & $p$ \\
\hline SOD1 (ng/mL) & $38.65 \pm 11.21$ & $47.49 \pm 13.76$ & 0.0575 \\
\hline SOD1 (ng/mg total protein) & $0.328 \pm 0.133$ & $0.393 \pm 0.106$ & 0.1275 \\
\hline SOD2 (ng/mL) & $\{24.71 ; 29.83 ; 34.94\}$ & $\{17.84 ; 22.22 ; 29.67\}$ & 0.0697 \\
\hline SOD2 (ng/mg total protein) & $\{0.183 ; 0.250 ; 0.298\}$ & $\{0.147 ; 0.187 ; 0.273\}$ & 0.2968 \\
\hline SOD3 $(\mathrm{ng} / \mathrm{mL})$ & $27.09 \pm 8.04$ & $27.46 \pm 6.37$ & 0.8857 \\
\hline SOD3 (ng/mg total protein) & $0.222 \pm 0.083$ & $0.240 \pm 0.072$ & 0.4982 \\
\hline $\mathrm{SOD}(\mathrm{U} / \mathrm{L})$ & $2157 \pm 611$ & $2262 \pm 330$ & 0.5392 \\
\hline SOD (U/g total protein) & $17.42 \pm 6.13$ & $19.07 \pm 3.90$ & 0.3559 \\
\hline SOD (U/mg SODs) & $22.84 \pm 7.39$ & $21.51 \pm 5.81$ & 0.5601 \\
\hline $\mathrm{Cu}, \mathrm{Zn}-\mathrm{SOD}(\mathrm{U} / \mathrm{L})$ & $\{530 ; 1050 ; 1301\}$ & $\{580 ; 795 ; 1071\}$ & 0.4827 \\
\hline $\mathrm{Cu}, \mathrm{Zn}-\mathrm{SOD}$ (U/g total protein) & $\{4.35 ; 8.98 ; 10.77\}$ & $\{4.79 ; 6.31 ; 9.40\}$ & 0.5904 \\
\hline Cu,Zn-SOD (U/mg SOD1+SOD3) & $13.80 \pm 5.15$ & $\mathbf{1 0 . 5 8} \pm 4.68$ & 0.0648 \\
\hline Cu,Zn-SOD (\% SOD activity) & $41.04 \pm 12.11$ & $36.97 \pm 11.61$ & 0.3181 \\
\hline${ }^{*} \mathrm{Mn}-\mathrm{SOD}(\mathrm{U} / \mathrm{L})$ & $\mathbf{1 1 0 5} \pm 979$ & $\mathbf{1 3 7 7} \pm 296$ & 0.0015 \\
\hline${ }^{*} \mathrm{Mn}-\mathrm{SOD}$ (U/g total protein) & $8.90 \pm 1.88$ & $\mathbf{1 1 . 9 7} \pm 2.55$ & 0.0006 \\
\hline * Mn-SOD (U/mg SOD2) & $44.43 \pm 14.80$ & $59.76 \pm 19.18$ & 0.0145 \\
\hline TAC (mM UAE) & $\mathbf{0 . 3 6 4} \pm 0.043$ & $0.396 \pm 0.053$ & 0.0581 \\
\hline $\operatorname{MDA}(\mu \mathrm{mol} / \mathrm{L})$ & $6.04 \pm 1.08$ & $7.01 \pm 3.18$ & 0.2350 \\
\hline $\mathrm{Cu}(\mu \mathrm{g} / \mathrm{L})$ & $993 \pm 168$ & $983 \pm 94$ & 0.8293 \\
\hline $\mathrm{Zn}(\mu \mathrm{g} / \mathrm{L})$ & $1038 \pm 132$ & $1022 \pm 90$ & 0.6960 \\
\hline $\mathrm{Zn} / \mathrm{Cu}$ & $1.03 \pm 0.17$ & $\mathbf{1 . 0 6} \pm 0.17$ & 0.5876 \\
\hline $\mathrm{Cd}(\mathrm{mg} / \mathrm{g} \mathrm{Hb})$ & $1.66 \pm 0.97$ & $2.04 \pm 1.14$ & 0.3178 \\
\hline
\end{tabular}

Values shown as: mean value \pm standard deviation and $\left\{1\right.$ st quartile; median value; 3 rd quartile\}. ${ }^{*}$ significant difference (mean values).

The analysis in context of exposition to cigarette smoke was performed regardless of sex due to relatively low count of exposed individuals. In both the non-obese (Table 4) and obese (Table 5), higher cadmium concentration $(p<0.0001)$ was found in individuals exposed to cigarette smoke, compared to the non-exposed. Interesting observations regarding the activity of SODs were made in the control group. In that group, the exposed were characterized of higher Mn-SOD activity $(p \approx 0.0146 ; p \approx 0.0419$ in case of Mn-SOD (U/L), Mn-SOD (U/g total protein), respectively) and markedly (approximately $49 \%$ ), but insignificantly $(p \approx 0.0592$ ) lower contribution of $\mathrm{Cu}, \mathrm{Zn}-\mathrm{SOD}$ to the total SOD activity pool. The values of the rest of analyzed parameters seemed unaffected by the exposition status.

Table 4. Values of selected pro- and antioxidative parameters, and concentration of selected metals, in context of exposition to cigarette smoke, in non-obese individuals.

\begin{tabular}{|c|c|c|c|}
\hline Parameter & $\begin{array}{l}\text { Non-Exposed }(n=41) \\
(24 \text { Women, } 17 \text { Men })\end{array}$ & $\begin{array}{c}\text { Exposed }(n=9) \\
(5 \text { Women, } 4 \text { Men) }\end{array}$ & $p$ \\
\hline SOD1 (ng/mL) & $\{18.86 ; 28.56 ; 37.42\}$ & $\{22.52 ; 30.24 ; 42.25\}$ & 0.7706 \\
\hline SOD1 (ng/mg total protein) & $\{0.160 ; \mathbf{0 . 2 1 1} ; 0.296\}$ & $\{0.212 ; 0.242 ; 0.379\}$ & 0.2788 \\
\hline SOD2 $(\mathrm{ng} / \mathrm{mL})$ & $\{21.73 ; 25.93 ; 33.21\}$ & $\{21.97 ; 28.30 ; 36.70\}$ & 0.7589 \\
\hline SOD2 (ng/mg total protein) & $\{0.169 ; 0.210 ; 0.268\}$ & $\{0.168 ; 0.224 ; 0.289\}$ & 0.6714 \\
\hline SOD3 $(\mathrm{ng} / \mathrm{mL})$ & $\{25.51 ; 29.57 ; 36.60\}$ & $\{13.42 ; 20.66 ; 38.77\}$ & 0.1879 \\
\hline SOD3 (ng/mg total protein) & $\{0.179 ; 0.243 ; 0.294\}$ & $\{0.103 ; 0.159 ; 0.286\}$ & 0.1779 \\
\hline $\mathrm{SOD}(\mathrm{U} / \mathrm{L})$ & $\{1569 ; 2290 ; 2522\}$ & $2087 ; 2603 ; 2803\}$ & 0.0910 \\
\hline SOD (U/g total protein) & $\{12.15 ; \mathbf{1 7 . 3 8} ; 20.71\}$ & $\{15.61 ; 20.52 ; 22.62\}$ & 0.1926 \\
\hline SOD (U/mg SODs) & $\{18.80 ; 24.32 ; 30.46\}$ & $\{17.84 ; 22.57 ; 32.37\}$ & 0.9650 \\
\hline $\mathrm{Cu}, \mathrm{Zn}-\mathrm{SOD}(\mathrm{U} / \mathrm{L})$ & $\{495 ; 854 ; 1264\}$ & $\{607 ; 847 ; 948\}$ & 0.6911 \\
\hline $\mathrm{Cu}, \mathrm{Zn}-\mathrm{SOD}$ (U/g total protein) & $\{4.05 ; 6.90 ; 10.29\}$ & $\{4.77 ; 6.26 ; 8.42\}$ & 0.6193 \\
\hline
\end{tabular}


Table 4. Cont.

\begin{tabular}{|c|c|c|c|}
\hline Parameter & $\begin{array}{l}\text { Non-Exposed }(n=41) \\
(24 \text { Women, } 17 \text { Men })\end{array}$ & $\begin{array}{c}\text { Exposed }(n=9) \\
(5 \text { Women, } 4 \text { Men) }\end{array}$ & $p$ \\
\hline $\mathrm{Cu}, \mathrm{Zn}-\mathrm{SOD}(\mathrm{U} / \mathrm{mg} \mathrm{SOD} 1+\mathrm{SOD} 3)$ & $\{9.21 ; 15.27 ; 19.07\}$ & $\{7.95 ; 14.90 ; 20.60\}$ & 0.9185 \\
\hline Cu,Zn-SOD (\% SOD activity) & $\{29.80 ; 45.53 ; 55.59\}$ & $\{24.57 ; 30.42 ; 33.13\}$ & 0.0592 \\
\hline ** Mn-SOD (U/L) & $\{1004 ; 1199 ; 1591\}$ & $\{1326 ; 1555 ; 2014\}$ & 0.0146 \\
\hline${ }^{* *}$ Mn-SOD (U/g total protein) & $\{6.92 ; 9.97 ; 11.66\}$ & $\{11.05 ; \mathbf{1 1 . 6 3} ; 15.76\}$ & 0.0419 \\
\hline Mn-SOD (U/mg SOD2) & $\{34.94 ; 42.81 ; 65.51\}$ & $\{33.32 ; 59.06 ; 65.93\}$ & 0.6929 \\
\hline TAC (mM UAE) & $\{0.249 ; 0.295 ; 0.354\}$ & $\{0.287 ; 0.304 ; 0.347\}$ & 0.5321 \\
\hline $\operatorname{MDA}(\mu \mathrm{mol} / \mathrm{L})$ & $\{3.86 ; 5.02 ; 6.18\}$ & $\{4.40 ; 5.19 ; 5.23\}$ & 0.9683 \\
\hline $\mathrm{Cu}(\mu \mathrm{g} / \mathrm{L})$ & $1014 \pm 170$ & $1075 \pm 90$ & 0.3597 \\
\hline $\mathrm{Zn}(\mu \mathrm{g} / \mathrm{L})$ & $951 \pm 111$ & $979 \pm 103$ & 0.5348 \\
\hline $\mathrm{Zn} / \mathrm{Cu}$ & $0.95 \pm 0.14$ & $0.95 \pm 0.21$ & 0.9811 \\
\hline ** Cd (mg/g Hb) & $\{1.09 ; 1.93 ; 2.58\}$ & $\{5.32 ; 6.30 ; 12.12\}$ & $<0.0001$ \\
\hline
\end{tabular}

Values shown as: mean value \pm standard deviation and $\{1$ st quartile; median value; 3 rd quartile $\} .{ }^{* *}$ significant difference (median values).

Table 5. Values of selected pro- and antioxidative parameters, and concentration of selected metals, in context of exposition to cigarette smoke, in obese individuals.

\begin{tabular}{|c|c|c|c|}
\hline Parameter & $\begin{array}{l}\text { Non-Exposed }(n=27) \\
(9 \text { Women, } 18 \text { Men) }\end{array}$ & $\begin{array}{c}\text { Exposed }(n=17) \\
(11 \text { Women, } 6 \text { Men })\end{array}$ & $p$ \\
\hline SOD1 (ng/mL) & $\{30.44 ; 37.10 ; 49.28\}$ & $\{27.20 ; 39.83 ; 45.23\}$ & 0.5469 \\
\hline SOD1 (ng/mg total protein) & $\{0.267 ; 0.328 ; 0.426\}$ & $\{0.206 ; 0.287 ; 0.393\}$ & 0.4778 \\
\hline SOD2 $(\mathrm{ng} / \mathrm{mL})$ & $\{19.69 ; 23.26 ; 32.70\}$ & $\{20.27 ; 24.52 ; 26.62\}$ & 0.6636 \\
\hline SOD2 (ng/mg total protein) & $\{0.151 ; 0.207 ; 0.279\}$ & $\{0.157 ; \mathbf{0 . 1 9 1} ; 0.228\}$ & 0.4478 \\
\hline SOD3 $(\mathrm{ng} / \mathrm{mL})$ & $\{25.64 ; 30.02 ; 33.84\}$ & $\{17.34 ; 27.96 ; 31.34\}$ & 0.1649 \\
\hline SOD3 (ng/mg total protein) & $\{0.202 ; 0.272 ; 0.296\}$ & $\{0.142 ; 0.243 ; 0.277\}$ & 0.0853 \\
\hline $\mathrm{SOD}(\mathrm{U} / \mathrm{L})$ & $\{1971 ; 2187 ; 2398\}$ & $\{1723 ; 2223 ; 2477\}$ & 1.000 \\
\hline SOD (U/g total protein) & $\{15.31 ; \mathbf{1 7 . 5 5} ; 20.78\}$ & $\{13.75 ; \mathbf{1 8 . 5 0} ; 20.47\}$ & 0.8763 \\
\hline SOD (U/mg SODs) & $\{17.95 ; 21.24 ; 25.63\}$ & $\{20.11 ; 22.95 ; 25.73\}$ & 0.3488 \\
\hline $\mathrm{Cu}, \mathrm{Zn}-\mathrm{SOD}(\mathrm{U} / \mathrm{L})$ & $\{580 ; 771 ; 1068\}$ & $\{652 ; 756 ; 852\}$ & 0.6540 \\
\hline $\mathrm{Cu}, \mathrm{Zn}-\mathrm{SOD}$ (U/g total protein) & $\{5.04 ; 6.06 ; 8.90\}$ & $\{5.06 ; 5.79 ; 6.62\}$ & 0.4380 \\
\hline $\mathrm{Cu}, \mathrm{Zn}-\mathrm{SOD}$ (U/mg SOD1+SOD3) & $\{7.21 ; 9.62 ; 14.29\}$ & $\{8.92 ; 10.54 ; 13.68\}$ & 0.8763 \\
\hline Cu,Zn-SOD (\% SOD activity) & $\{29.94 ; 37.89 ; 42.81\}$ & $\{28.02 ; 34.31 ; 41.72\}$ & 0.3907 \\
\hline Mn-SOD (U/L) & $\{1105 ; \mathbf{1 4 3 1} ; 1495\}$ & $\{1111 ; 1460 ; 1686\}$ & 0.3625 \\
\hline Mn-SOD (U/g total protein) & $\{9.27 ; \mathbf{1 1 . 3 3} ; 13.28\}$ & $\{8.73 ; 12.07 ; 13.85\}$ & 0.9243 \\
\hline Mn-SOD (U/mg SOD2) & $\{37.26 ; 56.35 ; 72.68\}$ & $\{42.08 ; 63.77 ; 82.98\}$ & 0.4668 \\
\hline TAC (mM UAE) & $\{0.360 ; \mathbf{0 . 3 8 6} ; 0.426\}$ & $\{0.330 ; 0.351 ; 0.420\}$ & 0.8559 \\
\hline $\mathrm{MDA}(\mu \mathrm{mol} / \mathrm{L})$ & $\{5.83 ; 6.60 ; 9.93\}$ & $\{6.22 ; 6.72 ; 8.57\}$ & 0.3653 \\
\hline $\mathrm{Cu}(\mu \mathrm{g} / \mathrm{L})$ & $\mathbf{1 0 6 1} \pm 158$ & $1103 \pm 142$ & 0.3889 \\
\hline $\mathrm{Zn}(\mu \mathrm{g} / \mathrm{L})$ & $\{892 ; 967 ; 1075\}$ & $\{860 ; 932 ; 1016\}$ & 0.3947 \\
\hline $\mathrm{Zn} / \mathrm{Cu}$ & $0.96 \pm 0.22$ & $\mathbf{0 . 8 6} \pm 0.16$ & 0.1263 \\
\hline * Cd (mg/g Hb) & $2.45 \pm 1.37$ & $\mathbf{1 2 . 5 4} \pm 7.89$ & $<0.0001$ \\
\hline
\end{tabular}

Values shown as: mean value \pm standard deviation and $\left\{1\right.$ st quartile; median value; 3rd quartile\}. ${ }^{*}$ significant difference (mean values).

2.3. Selected Correlations between the Concentration/Activity of SODs and Concentration of Copper, Zinc, and Cadmium, and Other Parameters

Firstly, monotonic (Spearman) correlations between the concentration of copper, zinc, and cadmium, concentration/activity of SODs, TAC values, and MDA concentration, and age, parameters describing lipid and carbohydrate metabolism, insulin resistance, and inflammation were analyzed, as shown in Table 6. Concentration of copper was positively correlated with age, TChol, TG, LDL-Chol, and CRP concentration. Concentration of zinc was positively correlated with TG, LDL-Chol, and glucose concentration, and negatively correlated with HDL-Chol concentration. Cadmium concentration was positively correlated with age and body mass index (BMI). 
Concentration of SOD1 was positively correlated with TG, insulin concentration and HOMA-IR index, and negatively correlated with HDL-Chol concentration. Total SOD activity was negatively correlated with parameters elevated in obesity: TChol, TG, CRP, insulin concentration, BMI, and HOMA-IR indices. $\mathrm{Cu}, \mathrm{Zn}-\mathrm{SOD}$ activity was negatively correlated with age, TChol, LDL-Chol concentration, and BMI index. Mn-SOD activity was not significantly correlated with any of the mentioned parameters. The contribution of $\mathrm{Cu}, \mathrm{Zn}$-SOD to the total SOD activity pool was negatively correlated with age and LDL-Chol concentration, and positively with HDL-Chol concentration. Interestingly, the values of TAC and MDA concentration were both positively correlated with age, TG, CRP, glucose, insulin concentration, BMI index, and negatively correlated with HDL-Chol concentration. Additionally, TAC values, unlike MDA concentration, were positively correlated with HOMA-IR index.

Table 6. Spearman correlation matrix between selected variables, in the entire population sample $(n=94)$.

\begin{tabular}{|c|c|c|c|c|c|c|c|c|c|c|}
\hline Variable & $\begin{array}{c}\text { Age } \\
\text { (years) }\end{array}$ & $\begin{array}{l}\text { TChol } \\
\text { (mg/dL) }\end{array}$ & $\begin{array}{c}\text { TG } \\
(\mathrm{mg} / \mathrm{dL})\end{array}$ & $\begin{array}{l}\text { HDL-Chol } \\
\text { (mg/dL) }\end{array}$ & $\begin{array}{l}\text { LDL-Chol } \\
\text { (mg/dL) }\end{array}$ & $\begin{array}{c}\text { CRP } \\
(\mathrm{mg} / \mathrm{L})\end{array}$ & $\begin{array}{l}\text { Glucose } \\
(\mathrm{mmol} / \mathrm{L})\end{array}$ & $\begin{array}{l}\text { Insulin } \\
(\mathrm{mU} / \mathrm{L})\end{array}$ & BMI & HOMA-IR \\
\hline $\begin{array}{c}1-11 \\
\mathrm{Cu}(\mu \mathrm{g} / \mathrm{L})\end{array}$ & 0.25 & 0.48 & 0.25 & & 0.37 & 0.34 & & & & \\
\hline $\begin{array}{c}1-11 \mathrm{Zn} \\
(\mu \mathrm{g} / \mathrm{L})\end{array}$ & & & 0.28 & -0.42 & 0.24 & & 0.22 & & & \\
\hline $\begin{array}{c}1-11 \mathrm{Cd} \\
(\mathrm{mg} / \mathrm{g} \mathrm{Hb})\end{array}$ & 0.41 & & & & & & & & 0.23 & \\
\hline $\begin{array}{c}\text { 1-11 SOD1 } \\
(\mathrm{ng} / \mathrm{mL})\end{array}$ & & & 0.32 & -0.48 & & & & 0.28 & & 0.30 \\
\hline $\begin{array}{c}\text { 1-11 SOD2 } \\
\text { (ng/mL) }\end{array}$ & & & & & & & & & & \\
\hline $\begin{array}{c}\text { 1-11 SOD3 } \\
(\mathrm{ng} / \mathrm{mL})\end{array}$ & & & & & & & & & & \\
\hline $\begin{array}{c}\text { 1-11 SOD } \\
(\mathrm{U} / \mathrm{L})\end{array}$ & & -0.24 & -0.26 & & & -0.26 & & -0.31 & -0.21 & -0.28 \\
\hline $\begin{array}{c}1-11 \\
\mathrm{Cu}, \mathrm{Zn}-\mathrm{SOD} \\
(\mathrm{U} / \mathrm{L})\end{array}$ & -0.37 & -0.25 & & & -0.26 & & & & -0.26 & \\
\hline $\begin{array}{c}1-11 \\
\mathrm{Mn}-\mathrm{SOD} \\
(\mathrm{U} / \mathrm{L}) \\
\end{array}$ & & & & & & & & & & \\
\hline $\begin{array}{c}1-11 \\
\text { Cu,Zn-SOD } \\
\text { (\% SOD } \\
\text { activity) }\end{array}$ & -0.41 & & & 0.23 & -0.26 & & & & & \\
\hline $\begin{array}{c}\text { 1-11 TAC } \\
\text { (mM UAE) }\end{array}$ & 0.43 & & 0.30 & -0.37 & & 0.32 & 0.42 & 0.23 & 0.59 & 0.23 \\
\hline $\begin{array}{c}\text { 1-11 MDA } \\
(\mu \mathrm{mol} / \mathrm{L})\end{array}$ & 0.28 & & 0.23 & -0.33 & & 0.34 & 0.27 & 0.24 & 0.48 & \\
\hline
\end{tabular}

Significant correlations are colored, depending on direction (red-negative, green-positive). Color saturation depends on the magnitude of correlation ( $\varrho$ value).

Secondly, correlations between the concentration/activity of SODs, TAC values and concentration of MDA, copper, zinc, and cadmium were analyzed, as shown in Table 7. SOD1 concentration was negatively correlated with SOD2 concentration and positively correlated with SOD3 concentration, total SOD activity, $\mathrm{Cu}, \mathrm{Zn}$-SOD activity, contribution of $\mathrm{Cu}, \mathrm{Zn}-\mathrm{SOD}$ to the total SOD activity pool, and TAC values. SOD3 concentration, apart from the mentioned correlation with SOD1 concentration, was positively correlated with $\mathrm{Cu}, \mathrm{Zn}-\mathrm{SOD}$ activity and contribution of $\mathrm{Cu}, \mathrm{Zn}$-SOD to the total SOD activity pool. Interestingly, both the $\mathrm{Cu}, \mathrm{Zn}-\mathrm{SOD}$ activity and contribution of $\mathrm{Cu}, \mathrm{Zn}$-SOD to the total SOD activity pool were negatively correlated with MDA concentration. Moreover, TAC values were positively correlated with MDA concentration. Both TAC values and MDA concentration were positively correlated with zinc concentration. Surprisingly, no significant correlations were found between the concentration of zinc and concentration/activity of SODs, copper concentration was negatively correlated with total SOD activity and $\mathrm{Cu}, \mathrm{Zn}-\mathrm{SOD}$ activity, and cadmium concentration was positively correlated with Mn-SOD activity. 
Table 7. Spearman correlation matrix between selected variables, in the entire population sample $(n=94)$.

\begin{tabular}{|c|c|c|c|c|c|c|c|c|c|c|c|c|}
\hline Variable & $\begin{array}{c}\text { SOD1 } \\
\text { (ng/mL) }\end{array}$ & $\begin{array}{l}\text { SOD2 } \\
\text { (ng/mL) }\end{array}$ & $\begin{array}{l}\text { SOD3 } \\
\text { (ng/mL) }\end{array}$ & $\begin{array}{l}\text { SOD } \\
\text { (U/L) }\end{array}$ & $\begin{array}{l}\mathrm{Cu}, \mathrm{Zn}-\mathrm{SOD} \\
\text { (U/L) }\end{array}$ & $\begin{array}{c}\text { Mn-SOD } \\
\text { (U/L) }\end{array}$ & $\begin{array}{c}\text { Cu,Zn-SOD } \\
\text { (\% SOD } \\
\text { activity) }\end{array}$ & $\begin{array}{l}\text { TAC } \\
(\mathrm{mM} \\
\text { UAE) }\end{array}$ & $\begin{array}{l}\text { MDA } \\
(\mu \mathrm{mol} / \mathrm{L})\end{array}$ & $\begin{array}{c}\mathrm{Cu} \\
(\mu \mathrm{g} / \mathrm{L})\end{array}$ & $\begin{array}{l}\mathrm{Zn} \\
(\mu \mathrm{g} / \mathrm{L})\end{array}$ & $\begin{array}{c}\mathrm{Cd} \\
(\mathrm{mg} / \mathrm{g} \\
\mathrm{Hb})\end{array}$ \\
\hline $\begin{array}{c}1-13 \\
\text { SOD1 } \\
(\mathrm{ng} / \mathrm{mL})\end{array}$ & & -0.29 & 0.23 & 0.19 & 0.33 & & 0.24 & 0.36 & & & & \\
\hline $\begin{array}{c}\text { 1-13 SOD2 } \\
(\mathrm{ng} / \mathrm{mL})\end{array}$ & -0.29 & & & & & & & & & & & \\
\hline $\begin{array}{c}\text { 1-13 SOD3 } \\
(\mathrm{ng} / \mathrm{mL})\end{array}$ & 0.23 & & & & 0.19 & & 0.24 & & & & & \\
\hline $\begin{array}{c}1-13 \text { SOD } \\
(\mathrm{U} / \mathrm{L})\end{array}$ & 0.19 & & & & 0.66 & 0.64 & & & & -0.24 & & \\
\hline $\begin{array}{c}1-13 \\
\mathrm{Cu}, \mathrm{Zn}-\mathrm{SOD} \\
(\mathrm{U} / \mathrm{L}) \\
\end{array}$ & 0.33 & & 0.19 & 0.66 & & & 0.78 & & -0.31 & & & \\
\hline $\begin{array}{c}1-13 \\
\mathrm{Mn}-\mathrm{SOD} \\
(\mathrm{U} / \mathrm{L})\end{array}$ & & & & 0.64 & & & -0.55 & & & -0.30 & & 0.22 \\
\hline $\begin{array}{c}1-13 \\
\text { Cu,Zn-SOD } \\
\text { (\% SOD } \\
\text { activity) } \\
\end{array}$ & 0.24 & & 0.24 & & 0.78 & -0.55 & & & -0.28 & & & \\
\hline $\begin{array}{c}\text { 1-13 TAC } \\
\text { (mM UAE) }\end{array}$ & 0.36 & & & & & & & & 0.33 & & 0.22 & \\
\hline $\begin{array}{l}\text { 1-13 MDA } \\
(\mu \mathrm{mol} / \mathrm{L})\end{array}$ & & & & & -0.31 & & -0.28 & 0.33 & & & 0.24 & \\
\hline
\end{tabular}

Significant correlations are colored, depending on direction (red-negative, green-positive). Color saturation depends on the magnitude of correlation ( $\varrho$ value).

\subsection{Genotyping Results—Genotypic Frequency in Context of Obesity}

Contingency tables (Table 8 ) showed no significant differences in genotype frequency, between the obese and non-obese, in SNPs: $r$ s2234694 ( $p \approx 0.5807)$, rs5746105 ( $p \approx 0.2985)$, $\operatorname{rs927450~(~} p \approx 0.8364)$, rs8192287 ( $p=1.0000)$. Regarding rs 2234694 , over $80 \%$ of individuals were of $\mathrm{A} / \mathrm{A}$ genotype regardless of obesity status. The $\mathrm{T}$ allele of rs 5746105 was detected in over $80 \%$ of individuals regardless of the obesity status and the $\mathrm{C} / \mathrm{C}$ genotype was slightly more frequent in obese $(15.91 \%)$, compared to the non-obese $(6.00 \%)$. The $\mathrm{T}$ allele of rs 927450 was found in over $70 \%$ of individuals regardless of obesity status and $24.00 \%$ and $20.45 \%$ of individuals were of C/C genotype in the control and the obese group, respectively. The SNP rs8192287 was ruled out of analysis, as in both control and obese groups all of individuals were of $G / G$ genotype.

Table 8. Contingency table of genotype distribution of the selected single nucleotide polymorphisms (SNPs), in context of obesity.

\begin{tabular}{|c|c|c|c|c|}
\hline SNP (Gene) & Genotype & Control Group $(n=50)$ & Obese Group $(n=44)$ & $p$ \\
\hline \multirow{3}{*}{ rs2234694 (SOD1) } & $\mathrm{A} / \mathrm{A}$ & $43(42.02)$ & $36(36.98)$ & \multirow{3}{*}{0.5807} \\
\hline & $\mathrm{A} / \mathrm{C}$ & $7(7.98)$ & $8(7.02)$ & \\
\hline & $\mathrm{C} / \mathrm{C}$ & $3(5.32)$ & $7(4.68)$ & \\
\hline \multirow[t]{3}{*}{ rs5746105 (SOD2) } & $\mathrm{C} / \mathrm{T}$ & $24(22.87)$ & $19(20.13)$ & \multirow[t]{2}{*}{0.2985} \\
\hline & $\mathrm{T} / \mathrm{T}$ & $23(21.81)$ & 18 (19.19) & \\
\hline & $\mathrm{C} / \mathrm{C}$ & $8(5.32)$ & $2(4.68)$ & \multirow{4}{*}{0.0015} \\
\hline \multirow[t]{3}{*}{ rs4880 (SOD2) } & $\mathrm{C} / \mathrm{T}$ & $31(38.30)$ & $41(33.70)$ & \\
\hline & $\mathrm{T} / \mathrm{T}$ & $11(6.38)$ & $1(5.62)$ & \\
\hline & $\mathrm{T} / \mathrm{T}$ & $16(15.43)$ & 13 (13.57) & \\
\hline \multirow[t]{2}{*}{ rs927450 (SOD2) } & $\mathrm{T} / \mathrm{C}$ & $22(23.40)$ & $22(20.60)$ & \multirow[t]{2}{*}{0.8364} \\
\hline & $\mathrm{C} / \mathrm{C}$ & 12 (11.17) & $9(9.83)$ & \\
\hline rs8192287 (SOD3) & $\mathrm{G} / \mathrm{G}$ & 50 & 44 & 1.0000 \\
\hline
\end{tabular}

Data shown as count (expected count). 
Significant $(p \approx 0.0015)$ difference in genotype frequency was found regarding SNP rs4880 (SOD2). Dominance of the $\mathrm{C} / \mathrm{T}$ genotype was observed in the obese group, compared to the control group $(93.18 \%$ vs. $62.00 \%)$. The remaining frequency was more less equally distributed between genotypes: $\mathrm{C} / \mathrm{C}$ and $\mathrm{T} / \mathrm{T}$. The difference in genotype frequency remained significant $(p \approx 0.0009)$ after merging the less frequent genotypes $(\mathrm{C} / \mathrm{C}$ and $\mathrm{T} / \mathrm{T})$ into one group.

\subsection{Values of SOD Concentration/Activity, TAC, and Concentration of MDA, in Context of Genotypic} Variability of the Chosen Single Nucleotide Polymorphisms in Genes: SOD1, SOD2, SOD3

Due to the lack of genotypes other than G/G, SNP rs8192287 (SOD3) was not included in this analysis. Two SNPs in this study, rs2234694 (SOD1) and rs4880 (SOD2), were found to be significantly associated with alterations in concentration/activity of SODs. The observations varied, depending on the obesity status. Descriptive statistics regarding the SNPs, which were not associated with any change in values of the anti(pro-)oxidative parameters assayed for in this study, is shown in Appendix A: Tables A1 and A2 (rs5746105), Tables A3 and A4 (rs927450).

Non-obese individuals (control group) of A/C genotype (rs2234694) showed approximately $45 \%$ lower SOD3 concentration in plasma $(p \approx 0.0165 ; p \approx 0.0304$ in case of SOD3 (ng/mL), SOD3 (ng/mg total protein), respectively) and higher MDA concentration $(p \approx 0.0423)$, compared to individuals of A/A genotype. Lower values of SOD1 concentration $(p \approx 0.0215 ; p \approx 0.0493$ in case of SOD1 (ng/mL), SOD1 (ng/mg total protein), respectively) and higher values of SOD2 concentration ( $p \approx 0.0460$ in case of both SOD2 $(\mathrm{ng} / \mathrm{mL})$ and SOD2 $(\mathrm{ng} / \mathrm{mg}$ total protein)) were found in obese individuals of $\mathrm{A} / \mathrm{C}$ genotype (rs2234694), compared to individuals of A/A genotype. The lack of difference in age, cadmium, and cotinine concentration and BMI between individuals of the two genotypes, in both the control and obese group indicate that age, exposition to cigarette smoke and uneven distribution of body fat (BMI index) are unlikely to be associated with the mentioned findings. Descriptive statistics regarding this SNP (rs2234694) are shown in Table 9 (non-obese) and Table 10 (obese).

Table 9. Values of selected pro- and antioxidative parameters, and concentration of selected metals, in context of genotypic variability of rs2234694 (SOD1), in non-obese individuals.

\begin{tabular}{|c|c|c|c|}
\hline Parameter & $\begin{array}{l}\text { A/A Genotype }(n=43) \\
\text { (26 Women, } 17 \text { Men) }\end{array}$ & $\begin{array}{c}\text { A/C Genotype }(n=7)(3 \\
\text { Women, } 4 \text { Men) }\end{array}$ & $p$ \\
\hline SOD1 (ng/mL) & $\{18.86 ; 29.15 ; 42.18\}$ & $\{24.08 ; 32.03 ; 33.91\}$ & 0.7427 \\
\hline SOD1 (ng/mg total protein) & $\{0.16 ; 0.23 ; 0.34\}$ & $\{0.18 ; 0.24 ; 0.30\}$ & 0.9782 \\
\hline SOD2 $(\mathrm{ng} / \mathrm{mL})$ & $\{22.32 ; 27.21 ; 34.69\}$ & $\{18.80 ; 21.97 ; 32.99\}$ & 0.2090 \\
\hline SOD2 (ng/mg total protein) & $\{0.17 ; 0.22 ; 0.27\}$ & $\{0.17 ; \mathbf{0 . 1 8} ; 0.28\}$ & 0.3544 \\
\hline ** SOD3 $(\mathrm{ng} / \mathrm{mL})$ & $\{25.51 ; 30.30 ; 38.00\}$ & $\{18.38 ; 20.66 ; 26.04\}$ & 0.0165 \\
\hline ** SOD3 (ng/mg total protein) & $\{0.18 ; 0.25 ; 0.30\}$ & $\{0.14 ; 0.16 ; 0.24\}$ & 0.0304 \\
\hline $\mathrm{SOD}(\mathrm{U} / \mathrm{L})$ & $\{1711 ; 2336 ; 2681\}$ & $\{1528 ; 2290 ; 2553\}$ & 0.5649 \\
\hline SOD (U/g total protein) & $\{13.20 ; 18.89 ; 20.71\}$ & $\{12.05 ; 19.47 ; 21.40\}$ & 0.8268 \\
\hline SOD (U/mg SODs) & $\{18.33 ; 23.81 ; 30.97\}$ & $\{22.80 ; 25.27 ; 34.03\}$ & 0.3391 \\
\hline $\mathrm{Cu}, \mathrm{Zn}-\mathrm{SOD}(\mathrm{U} / \mathrm{L})$ & $\{532 ; 847 ; 1252\}$ & $\{411 ; 1078 ; 1264\}$ & 0.9346 \\
\hline $\mathrm{Cu}, \mathrm{Zn}-\mathrm{SOD}$ (U/g total protein) & $\{4.23 ; 6.77 ; 9.94\}$ & $\{2.86 ; 8.42 ; 11.42\}$ & 0.9782 \\
\hline $\mathrm{Cu}, \mathrm{Zn}-\mathrm{SOD}$ (U/mg SOD1+SOD3) & $\{8.86 ; 14.79 ; 19.07\}$ & $\{9.51 ; 18.30 ; 26.34\}$ & 0.4908 \\
\hline Cu,Zn-SOD (\% SOD activity) & $\{27.92 ; 35.12 ; 54.55\}$ & $\{27.24 ; 43.27 ; 53.06\}$ & 1.0000 \\
\hline $\mathrm{Mn}-\mathrm{SOD}(\mathrm{U} / \mathrm{L})$ & $\{1004 ; 1261 ; 1676\}$ & $\{1098 ; 1145 ; 1223\}$ & 0.3495 \\
\hline Mn-SOD (U/g total protein) & $\{8.03 ; 10.07 ; 14.65\}$ & $\{8.14 ; 9.51 ; 11.05\}$ & 0.5465 \\
\hline Mn-SOD (U/mg SOD2) & $\{33.37 ; 42.81 ; 66.46\}$ & $\{33.91 ; 53.65 ; 65.93\}$ & 0.6714 \\
\hline TAC (mM UAE) & $\{0.25 ; 0.30 ; 0.35\}$ & $\{0.27 ; 0.35 ; 0.36\}$ & 0.7635 \\
\hline ** MDA $(\mu \mathrm{mol} / \mathrm{L})$ & $\{3.86 ; 5.02 ; 5.83\}$ & $\{4.48 ; 6.64 ; 7.96\}$ & 0.0423 \\
\hline $\mathrm{Cu}(\mu \mathrm{g} / \mathrm{L})$ & $\{921 ; 997 ; 1182\}$ & $\{853 ; 1059 ; 1241\}$ & 0.6027 \\
\hline $\mathrm{Zn}(\mu \mathrm{g} / \mathrm{L})$ & $\{846 ; 929 ; 1021\}$ & $\{902 ; 965 ; 1132\}$ & 0.3942 \\
\hline $\mathrm{Zn} / \mathrm{Cu}$ & $\{0.81 ; 0.91 ; 1.03\}$ & $\{0.83 ; 0.90 ; 1.07\}$ & 0.9129 \\
\hline
\end{tabular}


Table 9. Cont.

\begin{tabular}{|c|c|c|c|}
\hline Parameter & $\begin{array}{l}\text { A/A Genotype }(n=43) \\
(26 \text { Women, } 17 \text { Men })\end{array}$ & $\begin{array}{c}\text { A/C Genotype }(n=7)(3 \\
\text { Women, } 4 \text { Men) }\end{array}$ & $p$ \\
\hline $\mathrm{Cd}(\mathrm{mg} / \mathrm{g} \mathrm{Hb})$ & $\{1.62 ; 2.43 ; 3.51\}$ & $\{1.12 ; 1.49 ; 5.77\}$ & 0.5027 \\
\hline Cotinine $(\mathrm{ng} / \mathrm{mL})$ & $\{0.00 ; 0.00 ; 8.18\}$ & $\{0.00 ; \mathbf{0 . 0 0} ; 12.54\}$ & 0.5649 \\
\hline Age (years) & $\{24 ; 34 ; 47\}$ & $\{28 ; 38 ; 49\}$ & 0.5649 \\
\hline BMI & $\{21.36 ; 23.57 ; 26.79\}$ & $\{20.68 ; 21.72 ; 27.45\}$ & 0.7635 \\
\hline
\end{tabular}

Values shown as: mean value \pm standard deviation and $\{1$ st quartile; median value; 3 rd quartile $\} .{ }^{* *}$ significant difference (median values).

Table 10. Values of selected pro- and antioxidative parameters, and concentration of selected metals, in context of genotypic variability of rs2234694 (SOD1), in obese individuals.

\begin{tabular}{|c|c|c|c|}
\hline Parameter & $\begin{array}{l}\text { A/A Genotype }(n=36) \\
(15 \text { Women, } 21 \text { Men })\end{array}$ & $\begin{array}{c}\text { A/C Genotype }(n=8)(5 \\
\text { Men, } 3 \text { Women) }\end{array}$ & $p$ \\
\hline ** SOD1 (ng/mL) & $\{31.85 ; 41.92 ; 51.50\}$ & $\{23.06 ; 29.06 ; 37.58\}$ & 0.0215 \\
\hline ** SOD1 (ng/mg total protein) & $\{0.27 ; 0.36 ; 0.43\}$ & $\{0.16 ; 0.25 ; 0.34\}$ & 0.0498 \\
\hline ** SOD2 $(\mathrm{ng} / \mathrm{mL})$ & $\{18.70 ; 22.63 ; 28.03\}$ & $\{24.01 ; 27.17 ; 35.76\}$ & 0.0460 \\
\hline ** SOD2 (ng/mg total protein) & $\{0.15 ; 0.19 ; 0.25\}$ & $\{0.20 ; 0.23 ; 0.30\}$ & 0.0460 \\
\hline SOD3 (ng/mL) & $\{23.40 ; 30.02 ; 33.56\}$ & $\{26.68 ; 27.75 ; 30.84\}$ & 0.5095 \\
\hline SOD3 (ng/mg total protein) & $\{0.18 ; 0.26 ; 0.29\}$ & $\{0.19 ; 0.27 ; 0.29\}$ & 0.9390 \\
\hline $\mathrm{SOD}(\mathrm{U} / \mathrm{L})$ & $\{1953 ; 2198 ; 2477\}$ & $\{1610 ; 2031 ; 2332\}$ & 0.4590 \\
\hline SOD (U/g total protein) & $\{15.31 ; \mathbf{1 8 . 3 4} ; 20.28\}$ & $\{12.17 ; 15.98 ; 22.80\}$ & 0.6944 \\
\hline SOD (U/mg SODs) & $\{18.03 ; 21.98 ; 25.73\}$ & $\{20.82 ; 21.74 ; 24.06\}$ & 0.7895 \\
\hline $\mathrm{Cu}, \mathrm{Zn}-\mathrm{SOD}(\mathrm{U} / \mathrm{L})$ & $\{610 ; 764 ; 886\}$ & $\{390 ; 713 ; 1002\}$ & 0.7895 \\
\hline $\mathrm{Cu}, \mathrm{Zn}-\mathrm{SOD}$ (U/g total protein) & $\{5.18 ; 6.05 ; 7.65\}$ & $\{3.78 ; 5.54 ; 8.07\}$ & 0.6713 \\
\hline $\mathrm{Cu}, \mathrm{Zn}-\mathrm{SOD}(\mathrm{U} / \mathrm{mg} \mathrm{SOD} 1+\mathrm{SOD} 3)$ & $\{7.86 ; 10.24 ; 13.68\}$ & $\{8.03 ; 12.99 ; 17.43\}$ & 0.3195 \\
\hline Cu,Zn-SOD (\% SOD activity) & $\{29.47 ; 34.45 ; 41.49\}$ & $\{22.67 ; 38.93 ; 50.30\}$ & 0.7654 \\
\hline $\mathrm{Mn}-\mathrm{SOD}(\mathrm{U} / \mathrm{L})$ & $\{1174 ; 1441 ; 1582\}$ & $\{886 ; 1096 ; 1551\}$ & 0.2477 \\
\hline Mn-SOD (U/g total protein) & $\{9.48 ; 11.45 ; 13.37\}$ & $\{7.14 ; 10.13 ; 14.10\}$ & 0.5187 \\
\hline Mn-SOD (U/mg SOD2) & $\{41.91 ; \mathbf{5 9 . 5 6} ; 75.75\}$ & $\{24.17 ; 43.71 ; 60.09\}$ & 0.1385 \\
\hline TAC (mM UAE) & $\{0.33 ; 0.39 ; 0.45\}$ & $\{0.34 ; 0.38 ; 0.44\}$ & 0.9390 \\
\hline $\operatorname{MDA}(\mu \mathrm{mol} / \mathrm{L})$ & $\{5.71 ; 6.82 ; 8.69\}$ & $\{6.16 ; 6.58 ; 9.25\}$ & 0.6869 \\
\hline $\mathrm{Cu}(\mu \mathrm{g} / \mathrm{L})$ & $\{945 ; 1056 ; 1154\}$ & $\{975 ; 1134 ; 1312\}$ & 0.2763 \\
\hline $\mathrm{Zn}(\mu \mathrm{g} / \mathrm{L})$ & $\{890 ; 989 ; 1074\}$ & $\{836 ; 908 ; 944\}$ & 0.0622 \\
\hline $\mathrm{Zn} / \mathrm{Cu}$ & $\{0.82 ; 0.94 ; 1.07\}$ & $\{0.72 ; 0.78 ; 0.89\}$ & 0.0699 \\
\hline $\mathrm{Cd}(\mathrm{mg} / \mathrm{g} \mathrm{Hb})$ & $\{1.59 ; 3.92 ; 7.32\}$ & $\{1.70 ; 3.43 ; 6.15\}$ & 0.9860 \\
\hline Cotinine $(\mathrm{ng} / \mathrm{mL})$ & $\{0.00 ; \mathbf{0 . 0 0} ; 15.18\}$ & $\{0.00 ; 0.00 ; 13.11\}$ & 0.8423 \\
\hline Age (years) & $\{38 ; 46 ; 56\}$ & $\{39 ; 52 ; 58\}$ & 0.7540 \\
\hline BMI & $\{31.42 ; 32.29 ; 34.25\}$ & $\{31.57 ; 32.87 ; 34.99\}$ & 0.7090 \\
\hline
\end{tabular}

Values shown as: mean value \pm standard deviation and $\left\{1\right.$ st quartile; median value; 3 rd quartile\}. ${ }^{* *}$ - significant difference (median values).

As over $90 \%$ of obese individuals were of C/T genotype (rs4880), only the control group was included in the analysis in context of this genotype. Non-obese individuals of $\mathrm{T} / \mathrm{T}$ genotype showed lower SOD3 concentration in plasma $(p \approx 0.0663 ; p \approx 0.0486$ in case of SOD3 [ng/mL], SOD3 [ng/mg total protein], respectively), compared to individuals of $\mathrm{C} / \mathrm{C}$ or $\mathrm{C} / \mathrm{T}$ genotype. In the same (control) group, total SOD activity, $\mathrm{Cu}, \mathrm{Zn}-\mathrm{SOD}$ activity, and Mn-SOD activity were markedly lower in individuals of $\mathrm{C} / \mathrm{T}$ genotype. The type I error for comparisons in variables SOD [U/L], SOD [U/g total protein], $\mathrm{Cu}, \mathrm{Zn}$-SOD [U/L], Cu,Zn-SOD [U/g total protein], Mn-SOD [U/L], Mn-SOD [U/g total protein] were 0.0007, 0.0005, $0.0202,0.0189,0.0539$, and 0.0165 , respectively. No significant difference in age, cadmium, and cotinine concentration were found, indicating that age and exposition to smoke were unlikely to affect these findings. The difference in BMI values, being on the brink of statistical significance $(p \approx 0.0673)$ suggests a possible association of rs4880 (SOD2) with obesity. Descriptive statistics regarding this SNP (rs4880) are shown in Table 11. 
Table 11. Values of selected pro- and antioxidative parameters, and concentration of selected metals, in context of genotypic variability of rs4880 (SOD2), in non-obese individuals.

\begin{tabular}{|c|c|c|c|}
\hline Parameter & $\begin{array}{c}\text { C/C or C/T Genotype }(n=39) \\
(24 \text { Women, } 15 \text { Men })\end{array}$ & $\begin{array}{l}\text { T/T Genotype }(n=11) \\
(5 \text { Women, } 6 \text { Men) }\end{array}$ & $p$ \\
\hline SOD1 (ng/mL) & $\{19.78 ; 32.03 ; 42.16\}$ & $\{16.72 ; 28.56 ; 35.31\}$ & 0.5478 \\
\hline SOD1 (ng/mg total protein) & $\{0.17 ; 0.24 ; 0.35\}$ & $\{0.13 ; \mathbf{0 . 1 8} ; 0.27\}$ & 0.3187 \\
\hline SOD2 $(\mathrm{ng} / \mathrm{mL})$ & $\{22.12 ; 24.88 ; 33.44\}$ & $\{20.19 ; 28.11 ; 30.25\}$ & 0.7105 \\
\hline SOD2 (ng/mg total protein) & $\{0.17 ; 0.22 ; 0.28\}$ & $\{0.14 ; 0.21 ; 0.25\}$ & 0.5300 \\
\hline SOD3 $(\mathrm{ng} / \mathrm{mL})$ & $\{25.46 ; 30.02 ; 37.88\}$ & $\{15.70 ; 22.72 ; 29.21\}$ & 0.0663 \\
\hline ** SOD3 (ng/mg total protein) & $\{0.18 ; 0.24 ; 0.30\}$ & $\{0.11 ; 0.16 ; 0.25\}$ & 0.0486 \\
\hline$* * \operatorname{SOD}(\mathrm{U} / \mathrm{L})$ & $\{1865 ; 2359 ; 2685\}$ & $\{1411 ; 1642 ; 1899\}$ & 0.0007 \\
\hline ** SOD (U/g total protein) & $\{13.39 ; 20.02 ; 21.94\}$ & $\{9.52 ; \mathbf{1 1 . 1 8} ; 14.53\}$ & 0.0005 \\
\hline SOD (U/mg SODs) & $\{19.32 ; 23.86 ; 32.37\}$ & $\{17.20 ; 24.79 ; 26.62\}$ & 0.4338 \\
\hline ** Cu,Zn-SOD (U/L) & $\{532 ; 948 ; 1301\}$ & $\{411 ; 573 ; 642\}$ & 0.0202 \\
\hline ** Cu,Zn-SOD (U/g total protein) & $\{4.23 ; 8.42 ; 10.77\}$ & $\{2.68 ; 4.80 ; 5.19\}$ & 0.0189 \\
\hline $\mathrm{Cu}, \mathrm{Zn}-\mathrm{SOD}$ (U/mg SOD1+SOD3) & $\{10.36 ; 15.81 ; 20.02\}$ & $\{8.82 ; \mathbf{1 1 . 8 1} ; 18.17\}$ & 0.3618 \\
\hline Cu,Zn-SOD (\% SOD activity) & $\{29.80 ; 41.26 ; 54.55\}$ & $\{27.24 ; 31.25 ; 49.68\}$ & 0.5173 \\
\hline $\mathrm{Mn}-\mathrm{SOD}(\mathrm{U} / \mathrm{L})$ & $\{1033 ; \mathbf{1 3 2 6} ; 1885\}$ & $\{609 ; 1155 ; 1261\}$ & 0.0539 \\
\hline ** Mn-SOD (U/g total protein) & $\{8.81 ; \mathbf{1 0 . 6 1 ;} 15.06\}$ & $\{5.43 ; 8.60 ; 10.07\}$ & 0.0165 \\
\hline Mn-SOD (U/mg SOD2) & $\{35.97 ; 43.99 ; 65.93\}$ & $\{19.80 ; 40.36 ; 54.32\}$ & 0.3618 \\
\hline TAC (mM UAE) & $\{0.25 ; \mathbf{0 . 3 0} ; 0.35\}$ & $\{0.22 ; 0.30 ; 0.40\}$ & 0.4178 \\
\hline $\operatorname{MDA}(\mu \mathrm{mol} / \mathrm{L})$ & $\{3.86 ; 5.10 ; 5.83\}$ & $\{4.05 ; 5.40 ; 6.80\}$ & 0.3915 \\
\hline $\mathrm{Cu}(\mu \mathrm{g} / \mathrm{L})$ & $\{865 ; 995 ; 1184\}$ & $\{989 ; 1021 ; 1102\}$ & 0.2753 \\
\hline $\mathrm{Zn}(\mu \mathrm{g} / \mathrm{L})$ & $\{853 ; 929 ; 1020\}$ & $\{895 ; 992 ; 1132\}$ & 0.3540 \\
\hline $\mathrm{Zn} / \mathrm{Cu}$ & $\{0.81 ; 0.91 ; 1.06\}$ & $\{0.88 ; 0.91 ; 1.07\}$ & 0.9632 \\
\hline $\mathrm{Cd}(\mathrm{mg} / \mathrm{g} \mathrm{Hb})$ & $\{1.64 ; 2.43 ; 4.26\}$ & $\{1.03 ; \mathbf{2 . 1 8} ; 2.79\}$ & 0.2266 \\
\hline Cotinine (ng/mL) & $\{0.00 ; 0.00 ; 8.29\}$ & $\{0.00 ; 0.00 ; 0.00\}$ & 0.3302 \\
\hline Age (years) & $\{25 ; 33 ; 47\}$ & $\{27 ; 40 ; 49\}$ & 0.4178 \\
\hline BMI & $\{21.15 ; 22.83 ; 26.44\}$ & $\{23.32 ; 24.69 ; 27.44\}$ & 0.0673 \\
\hline
\end{tabular}

Values shown as: $\left\{1\right.$ st quartile; median value; 3 rd quartile\}. ${ }^{* *}$ — significant difference (median values).

2.6. Other Findings Regarding Genotypic Variability of Single Nucleotide Polymorphisms (SNPs): rs2234694 (SOD1), rs4880 (SOD2)

An unexpected observation was made regarding the genotypic variability of SNPs rs2234694 (SOD1) and rs4880 (SOD2), which, in this study, were associated with alterations in concentration/activity of SODs. Interestingly, the significant differences were found in parameters associated with carbohydrate metabolism and insulin resistance (Table 12). The obese of $\mathrm{A} / \mathrm{C}$ genotype (rs2234694) were characterized of over $70 \%$ lower median insulin concentration $(p \approx 0.0214)$ and over $80 \%$ lower median HOMA-IR index value $(p \approx 0.0218)$, compared to individuals of A/A genotype. The non-obese of $\mathrm{T} / \mathrm{T}$ genotype (rs4880) showed higher values of glucose concentration $(p \approx 0.0326)$, insulin concentration $(p \approx 0.0369)$ and HOMA-IR index $(p \approx 0.0306)$, compared to individuals of C/C or $\mathrm{C} / \mathrm{T}$ genotype, indicating a possible association of rs4880 (SOD2) with the state of obesity and insulin resistance. More thorough information on values of basic clinical parameters (lipidogram, glucose, insulin, CRP concentration, HOMA-IR index) can be found in Appendix A (Table A5). 
Table 12. The values of selected carbohydrate metabolism and insulin resistance parameters, in context of genotypic variability of rs2234694 (SOD1) and rs4880 (SOD2).

\begin{tabular}{|c|c|c|c|}
\hline \multicolumn{4}{|c|}{ rs2234694 (SOD1), Obese Group } \\
\hline Parameter & A/A Genotype $(n=36)$ & A/C Genotype $(n=8)$ & $p$ \\
\hline Glucose (mmol/L) & $\{4.94 ; 5.20 ; 5.61\}$ & $\{4.67 ; 4.92 ; 5.28\}$ & 0.2502 \\
\hline ** Insulin (mU/L) & $\{10.00 ; 14.60 ; 19.30\}$ & $\{7.80 ; 8.40 ; 9.90\}$ & 0.0214 \\
\hline ** HOMA-IR & $\{2.25 ; 3.21 ; 4.91\}$ & $\{1.62 ; 1.75 ; 1.85\}$ & 0.0218 \\
\hline \multicolumn{4}{|c|}{ rs4880 (SOD2), Non-Obese Group } \\
\hline Parameter & C/C or C/T Genotype $(n=39)$ & T/T Genotype $(n=11)$ & $p$ \\
\hline ** Glucose (mmol/L) & $\{4.50 ; 4.64 ; 4.89\}$ & $\{4.72 ; 4.94 ; 5.17\}$ & 0.0326 \\
\hline ** Insulin (mU/L) & $\{4.70 ; 6.60 ; 8.20\}$ & $\{6.50 ; 10.00 ; 15.20\}$ & 0.0369 \\
\hline${ }^{* *}$ HOMA & $\{0.97 ; 1.35 ; 1.84\}$ & $\{1.28 ; 2.25 ; 3.56\}$ & 0.0306 \\
\hline
\end{tabular}

Values shown as: $\{1$ st quartile; median value; 3 rd quartile $\} .{ }^{* *}$ significant difference (median values).

\section{Discussion}

The first issue to be addressed in this discussion is the proper a priori classification of individuals into study groups and the identity of the results of basic parameters used in laboratory diagnostics. The obese group was characterized of higher TG, CRP, glucose, insulin, BMI and HOMA-IR indices, and lower HDL-Chol concentration, compared to the non-obese group, which accounts for the lack of discrepancy between the observed characteristics of both groups and the "typical" clinical picture of obesity: insulin resistance, improper distribution of body fat, dyslipidemia and the state of meta-inflammation. However, virtually, it is possible that the difference in age between the control and obese group might have had some impact on the values of pro- and antioxidative parameters presented in this study. Such parameters could have been total SOD activity and concentration of MDA, which, according to the literature, are positively correlated with age [48-50]. Another such parameter is $\mathrm{Cu}, \mathrm{Zn}$-SOD activity and TAC, which were shown to be negatively correlated with age [51,52]. Moreover, Paik et al. [53] showed that the activity of $\mathrm{Cu}, \mathrm{Zn}$-SOD could be a marker of zinc supply with diet. Due to this fact, the amount of zinc in the diet might have had some impact on the values of $\mathrm{Cu}, \mathrm{Zn}-\mathrm{SOD}$. Therefore, the issues covered in this study should be further investigated in future research.

The first factor taken into consideration in this study was the intersex variability of the values of SOD concentration, TAC values, the concentration of MDA and metals: copper, zinc, and cadmium. Men were shown to be characterized by over 1.5 higher concentration of SOD1 and lower SOD3 concentration in plasma, compared to women. The values of TAC and MDA concentration were higher in men. Such an observation seems reliable, since the alterations in TAC are associated with the variability in the concentration of sex hormones [54]. Observations regarding intersexual variability in the concentration of the studied metals are consistent with the results of scientific studies of other authors, in which men have higher zinc concentration, the ratio of zinc to copper, and lower copper, serum, and cadmium in whole blood, in relation to women [55-58]. The literature also indicates increased accumulation of cadmium by women in relation to men [59-61], which coincides with the observations made in this study.

Secondly, two factors, obesity and exposition to cigarette smoke, were taken into consideration. Regardless of sex, markedly increased TAC values were observed in the obese group, although the $p$ value in men was slightly above the $\alpha(p \approx 0.058)$. The increase in TAC values could be due to an increase in the concentration of low-molecular-weight antioxidants, such as uric acid, tocopherols, glutathione, and ascorbic acid. The concentration of low-molecular-weight antioxidants was not assayed for in this study. Therefore, the origin of the increase in TAC values observed in this study should be investigated in future research. Interestingly the alterations in values of 
SOD concentration/activity and the concentration of MDA and metals depended on sex. In women, no difference in SOD concentration/activity was found between the obese and non-obese, although increased MDA, copper concentration, and decreased zinc-to-copper ratio were found in the obese. Conversely, obese men were characterized of increased activity and specific activity of Mn-SOD and no significant differences were observed in values of TAC, MDA, copper concentration, and zinc-to-copper ratio between the obese and non-obese. In both men and women, cadmium concentration was elevated in the obese, although in women this increase was more prominent (therefore statistically significant). Interestingly, in men, SOD1 concentration was increased, and SOD2 concentration was decreased in the obese, compared to the non-obese, although this observation should be investigated further as the $p$-values were ambiguous $(p \approx 0.0575$ and $p \approx 0.0697$ regarding SOD1 (ng/mL) and SOD2 $(\mathrm{ng} / \mathrm{mL})$, respectively). These findings may indicate that men and women adapt differently to the increased oxidative stress in state of obesity. Other research found in the literature shows a decrease in serum total SOD activity in the obese [62-64], an increase total SOD activity in the extracellular fluid [65] and blood [66,67] or no difference in total SOD activity between the obese and non-obese [68]. Decreased $\mathrm{Cu}, \mathrm{Zn}-\mathrm{SOD}$ activity was found in the erythrocytes of the obese [69]. The discrepancy between these results may stem not only from the difference in the type of studied specimen, but also from differences in age of the obese. Karaouzene et al. [70] showed that younger obese individuals were characterized of higher total SOD activity than the older obese individuals, compared to the control groups.

Interesting observations were made in context of exposition to cigarette smoke. In the non-obese, Mn-SOD activity was increased in the exposed. Moreover, the exposed were characterized of markedly (although not clearly significantly, $p \approx 0.0592$ ) decreased $\mathrm{Cu}, \mathrm{Zn}$-SOD contribution to the total SOD activity pool. In the obese, no such differences were observed. The decrease in $\mathrm{Cu}, \mathrm{Zn}-\mathrm{SOD}$ contribution to the total SOD activity pool may be due to inactivation of $\mathrm{Cu}, \mathrm{Zn}$-SOD by the xenobiotics of the cigarette smoke, such as cadmium — a well-known, specific Cu,Zn-SOD inhibitor [26,71] in this study-found to be of increased concentration in individuals exposed to cigarette smoke, regardless of obesity. Elevation of Mn-SOD activity may be an adaptive mechanism to increased oxidative stress, as proposed earlier. The discrepancy between the results obtained in the obese and non-obese, in this context, may indicate a loss of the ability to adapt to cigarette smoke via the increase of Mn-SOD activity in plasma of the obese. The topic of the association between Mn-SOD activity and exposition to cigarette smoke should be further investigated.

Correlations between the studied parameters were analyzed in an attempt to explain some of the observations made in this study. Concentration of copper, cadmium, TAC, and MDA increased with age. The decrease of $\mathrm{Cu}, \mathrm{Zn}$-SOD activity with age may be due to inhibition by increasing concentration of cadmium. Increasing glycemia and dyslipidemia observed in obesity were associated with intensification of the process of lipid peroxidation, as shown by the increase of MDA concentration in plasma. As TAC correlated with the same parameters (Table 6) as MDA, and additionally, positively with HOMA-IR, it could be assumed that TAC plays an important role in adaptation to increased oxidative stress due to hyperglycemia, dyslipidemia, and insulin resistance, observed in obesity. Therefore, low-molecular-weight antioxidants, presumably, could support the antioxidative system in the state of increasing inflammation and improper distribution of body fat, in which the total SOD activity is decreased (negative correlation of CRP with total SOD activity, negative correlation of BMI with total SOD activity and Cu,Zn-SOD activity). The lack of correlation between SOD activity and TAC values supports the thesis that enzymatic antioxidants do not significantly contribute to TAC values [72-74]. Interestingly, HOMA-IR correlated positively with SOD1 concentration, although no significant correlation was found between HOMA-IR and $\mathrm{Cu}, \mathrm{Zn}-\mathrm{SOD}$ activity. This could be due to lower contribution of SOD1 to Cu,Zn-SOD activity, although the correlation between SOD1 and $\mathrm{Cu}, \mathrm{Zn}$-SOD activity, analyzed in this study, was stronger than the correlation between SOD3 and $\mathrm{Cu}, \mathrm{Zn}-\mathrm{SOD}$ activity. The correlation between zinc concentration with TAC values and MDA concentration could be due to covariance between TG and HDL-Chol 
concentration and zinc concentration, TAC values, and MDA concentration. Therefore, it is likely to be biased. The positive correlation between the concentration of cadmium and Mn-SOD activity accounts for the aforementioned thesis that Mn-SOD plays an important role in the process of adaptation to cigarette smoke. The correlations between SOD1 concentration and SOD3 (positive) and SOD2 (negative) indicate that the three SOD isozymes may be a part of a mechanism which controls their concentration. This interesting issue, covering the origin of the occurrence of intracellular SOD isozymes (SOD1, SOD2) in the plasma and the interaction between the three isozymes, should be further investigated.

All of the aforementioned findings were the basis for analyzing the alterations in concentration/activity of SOD isozymes in context of selected SNPs of genes: SOD1, SOD2, SOD3. One of the selected SNPs (rs8192287, SOD3) could not be further analyzed in this study due to the fact that all individuals were of $\mathrm{G} / \mathrm{G}$ genotype. Rare frequency of genotypes other than $\mathrm{G} / \mathrm{G}$ is highly probable, since the data from the dbSNP database (National Center for Biotechnology Information) account for rare $(\approx 5.0 \%)$ occurrence of the $\mathrm{T}$ allele in the European countries.

Interestingly, the C/T genotype of another SNP, the rs4880 (SOD2), occurred in more than $90 \%$ of the obese group, indicating that this polymorphism might be a hereditary factor for developing obesity. This assumption is supported by the study of Montano et al. [47], in which one of the rs4880 genotypes, Val/Val (T/T), was associated with approximately two-fold increased chance of developing obesity in the Brazilian population. However, compared to our study, a different method, logistic regression, was used, allowing to control the impact of other factors, such as dyslipidemia, and values of systolic blood pressure on the obtained results, and individuals of BMI $>25$ were removed from the control group. Logistic regression was not used in this study as only 3 obese individuals out of 44 were of genotype other than C/T. In this case, the maximum likelihood estimation used in logistic regression would be highly biased. In this study, the genotype variability of rs 4880 has been found to be associated with alterations in SOD activity, although the statistical analyses could only be performed in the non-obese group, due to the aforementioned dominance of the $\mathrm{C} / \mathrm{T}$ genotype in the obese group. In the non-obese, the individuals of the $\mathrm{T} / \mathrm{T}$ genotype were of drastically decreased total SOD activity, $\mathrm{Cu}, \mathrm{Zn}$-SOD activity, and Mn-SOD activity in plasma, compared to the individuals of other genotypes ( $C / C$ or $C / T)$. These observations support the findings of research by Flekac et al. [40], in which the lowest serum total SOD activity was observed in individuals of T/T genotype. It is known that rs4880 is connected with Val16Ala substitution in the mitochondrial targeting sequence of SOD2 [45]. However, it has not been clarified how this substitution may affect the activity of both $\mathrm{Cu}, \mathrm{Zn}$-SOD and Mn-SOD in extracellular fluids. Surprisingly, in the non-obese group, the individuals of $\mathrm{T} / \mathrm{T}$ genotype were characterized by significantly higher concentration of glucose and insulin and higher HOMA-IR values, supporting the theory of association between the genotypic variability of rs4880 and increased susceptibility to obesity due to a decrease in the activity of SOD isozymes, leading to increased susceptibility to oxidative damage in tissues, such as $\beta$ pancreatic cells and altering the response of these cells to the glycemic status.

Other interesting observations were made in the context of genotypic variability of rs 2234694 (SOD1). Non-obese individuals of the rare (frequency $\approx 4.5 \%$, according to the dsSNP) $\mathrm{A} / \mathrm{C}$ genotype showed decreased SOD3 concentration, whereas the obese individuals of A/C genotype were characterized of lower SOD1 concentration and higher SOD2 concentration, compared to the individuals of the A/A genotype. This observation is rather surprising, since rs 2234694 is located in the intragenic region of SOD1. The alterations in SOD2 and SOD3 concentration may be associated with the aforementioned covariance between SOD isozyme concentration in plasma, shown in this study. No significant alterations in the activity of SOD isozymes were observed in the context of rs2234694. It has previously been reported that the C/C genotype (not detected in this study) is associated with lower total SOD activity, but in the diabetic individuals. Since the concentration of SOD isozymes was not assayed for in that study, it is impossible to discuss these observations any further [40], in context of this study. Interestingly, although the distribution of A/A and A/C genotypes, in this study, did not 
differ in context of obesity, the obese of A/C genotype showed over 1.5-fold lower concentration of insulin and values of HOMA-IR. Perhaps the association of this SNP (rs2234694) with insulin resistance and susceptibility to type two diabetes should be investigated in future research.

\section{Materials and Methods}

\subsection{Material}

The research was carried out in full blood, serum, plasma, and DNA, isolated from full blood. Full blood was obtained from the biobank of the Polish Center for Technology Development (Wrocław, Poland). Full blood was collected with use of Vacutainer set (Becton Dickinson), according to the standard diagnostic procedure. The samples for obtaining the serum were collected in test tubes containing a coagulation activator (BD Vacutainer, cat. no. 368815) and the samples for obtaining the plasma and isolating DNA were collected in test tubes containing $\mathrm{K}_{2}$ EDTA (BD Vacutainer, cat. no. 367864). Serum or plasma was obtained by centrifuging the full blood in $2000 \mathrm{~g}$, for $15 \mathrm{~min}$. The material was portioned and kept in $-80^{\circ} \mathrm{C}$, in Eppendorf test tubes (Eppendorf, cat. no. 0030102.002). The research project has been approved by the Bioethical Committee of Wroclaw Medical University (opinion no. KB 256/2019).

\subsection{Characteristics of the Population Sample and the Criteria for Exclusion from the Study and Division into Groups}

The exclusion criteria were inflammatory and metabolic diseases (other than obesity), cardiovascular diseases tumor diseases and simultaneous treatment with more than two types of drugs, regardless of their mechanism of action. The individuals from whom the specimen was collected were volunteers recruited by the Polish Center for Technology Development (Wroclaw, Poland). The specimen and the information regarding age, height and weight, diseases, treatment, and smoking status were collected on consent of the volunteers. The specimen was assayed for basic laboratory medicine parameters, such as: lipidogram, glucose concentration, insulin concentration, C-reactive protein concentration. HOMA-IR and BMI indices were calculated. The values of these parameters are shown in Table 13. All of the obtained biobanked samples were used in the study.

Table 13. The structure and characteristics of groups: control and obese.

\begin{tabular}{|c|c|c|c|}
\hline \multicolumn{4}{|c|}{ Structure of the Population Sample } \\
\hline Total count & 50 & \multicolumn{2}{|c|}{44} \\
\hline Sex & M: 21 & M: 24 & F: 20 \\
\hline Exposed to cigarette smoke & NO: 41 & NO: 27 & YES: 17 \\
\hline Sex (exposed) & M: 4 & M: 6 & $\mathrm{~F}: 11$ \\
\hline \multicolumn{4}{|c|}{ Characteristics of the Population Sample } \\
\hline Variable & Control Group & Obese Group & $p$ \\
\hline ** Age (years) & $\{25 ; 34 ; 47\}$ & $\{37 ; 47 ; 57\}$ & $<0.0001$ \\
\hline${ }^{* *} \mathrm{CRP}(\mathrm{mg} / \mathrm{L})$ & $\{0.33 ; 0.65 ; 1.10\}$ & $\{1.11 ; 1.41 ; 3.31\}$ & $<0.00001$ \\
\hline ** Glucose (mmol/L) & $\{4.50 ; 4.78 ; 4.94\}$ & $\{4.89 ; 5.17 ; 5.61\}$ & $<0.00001$ \\
\hline ** Insulin (mU/L) & $\{4.90 ; 6.90 ; 9.00\}$ & $\{9.50 ; 13.95 ; 18.00\}$ & $<0.00001$ \\
\hline ** BMI & $\{21.30 ; 23.48 ; 26.79\}$ & $\{31.51 ; 32.29 ; 34.25\}$ & $<0.00001$ \\
\hline ** HOMA-IR & $\{1.00 ; 1.49 ; 1.94\}$ & $\{1.85 ; 3.06 ; 4.23\}$ & $<0.00001$ \\
\hline
\end{tabular}

Values shown as: $\{1$ st quartile; median value; 3 rd quartile $\} .{ }^{* *}$ significant difference (median values). 
The main grouping factor was obesity, confirmed on the basis of recruitment information and the values of body mass index (BMI). The cut-off BMI value was 30, according to the WHO recommendations. The population sample consisted of 94 individuals, of which 50 individuals, aged 20-57 years (median value 34) were assigned to the control group and 44 individuals aged 24-75 years (median value 47) were assigned to the obese group. The structure of both these groups are shown in Table 13.

The secondary grouping criterion was exposition to cigarette smoke. It was highly probable that some individuals who were chronically exposed to cigarette smoke would not describe themselves as "smokers" during the recruitment process. Therefore, the classification was based on the cut-off value obtained in a logistic regression model, where the dependent variable was smoking declaration (based on the recruitment survey) and the explanatory variable was the concentration of cotinine, one of the main nicotine biotransformation end products, in serum:

$$
P\left(Y=1 \mid x_{1}, x_{2}, \ldots, x_{k}\right)=\frac{e^{(-3.248+0.273 x)}}{1+e^{(-3.248+0.273 x)}}
$$

where " $x$ " is the concentration of cotinine $(\mathrm{ng} / \mathrm{mL})$. The cut-off value of cotinine concentration, for exposition to cigarette smoke, was $10.6 \mathrm{ng} / \mathrm{mL}$, based on the Youden index (0.87) of the obtained ROC curve. This value lies within the cut-off value range $(10-20 \mathrm{ng} / \mathrm{mL})$ found in the literature [75-77].

\subsection{Methods}

The measurements were performed with use of SPECORD 40 (Analytik Jena, Jena, Germany) or Multiskan GO (ThermoFisher Scientific, Waltham, MA, USA) spectrophotometers, if not stated otherwise. The necessary incubations were performed in MC-01N (Omega Lab, Delhi, India) or StatFax 2200 (Awareness Technology Inc., Palm City, FL, USA).

4.3.1. Selected Markers of Nicotine Exposure, Carbohydrate, and Lipid Metabolism and Inflammation

Serum cotinine concentration was assayed with use of an ELISA kit (Calbiotech, cat. no. CO096D, El Cajon, CA, USA), utilizing polyclonal anti-human cotinine antibodies. Insulin concentration was determined in serum with use of an ELISA kit (Mercodia, cat. no. 10-1113-01, Uppsala, Sweden), utilizing murine monoclonal anti-human insulin antibodies. Serum glucose concentration was assayed for with use of a glucose oxidase kit (Biomaxima, cat. no. 1-033-0400, Lublin, Poland). A glicerol-3-phosphate oxidase method kit (Biomaxima, cat. no. 1-053-0200, Lublin, Poland) was used in serum triglyceride (TG) concentration assay.

Serum total cholesterol (TChol) concentration was determined with use of GPO/PAP kit (Biomaxima, cat. no. 1-023-0200, Lublin, Poland). Chylomicron, LDL and VLDL cholesterol fractions were precipitated (Biomaxima, cat. no. 1-030-0060, Lublin, Poland) and HDL cholesterol (HDL-Chol) was assayed for with use of the total cholesterol kit mentioned above. Since no triglyceride concentration values over $400 \mathrm{mg} / \mathrm{dL}$ were observed, the LDL cholesterol (LDL-Chol) concentration was determined with use of the Friedewald formula [78].

Serum C-reactive protein (CRP) concentration was assayed for with use of a turbidimetric kit, utilizing latex beads bound to anti-human CRP antibodies (Bio-Bas, cat. no. 48030).

\subsubsection{Concentration and Activity of Superoxide Dismutase Isozymes}

Superoxide dismutase (SOD) isozyme concentrations were assayed for in plasma with use of ELISA kits. SOD1 concentration kit (ThermoFisher Scientific, cat. no. BMS222, Waltham, MA, USA) utilized monoclonal anti-human SOD1 antibodies, whereas SOD2 (Biomatik, cat. no. 07502, Wilmington, DE, USA) and SOD3 (Biomatik, cat. no. 07504, Wilmington, DE, USA) concentration kits utilized polyclonal anti-human SOD2 (or SOD3) antibodies. 
Plasma SOD activity was determined with use of xanthine oxidase/tetrazolium salt method kit (Cayman Chemical, cat. no. 706002, Ann Arbor, MI, USA). Two types of activity were assayed for. Total SOD activity was the activity measured without presence of SOD inhibitors, whereas Mn-SOD (SOD2) activity was measured in presence of potassium cyanide (Merck, cat. no. 1.04967.0100, Kenilworth, NJ, USA), a specific Cu,Zn-SOD inhibitor $[79,80]$. As a result of optimization of the inhibition reaction, the following conditions were used: $25 \mathrm{~min}$ of preincubation of plasma with $3 \mathrm{mM}$ potassium cyanide in $25^{\circ} \mathrm{C}$, in volumetric ratio of $1: 1$. The absorbance values were read after $90 \mathrm{~min}$ after initializing the reaction by addition of xanthine oxidase. $\mathrm{Cu}, \mathrm{Zn}-\mathrm{SOD}$ activity, attributed to SOD1 and SOD3, was calculated by subtracting the values of Mn-SOD activity from the values of total SOD activity.

The values of SOD activity in this work are given in four variants: raw values $(\mathrm{U} / \mathrm{mL})$, values corrected by total protein concentration (U/g total protein), specific activity (U/mg SOD isozyme(s)), and contribution to the total SOD activity pool (\% total SOD activity). Total protein concentration was determined in plasma with use of micro-biuret method [81], in reference to the standard absorbance curve of BSA (Sigma Chemical, cat. no. A-2153, St. Louis, MI, USA).

\subsubsection{Total Antioxidative Capacity and Malondialdehyde Concentration}

Total antioxidative capacity (TAC) was determined in serum with use of a kit (Cell Biolabs, cat. no. STA-360, San Diego, CA, USA). Uric acid was used as a reference antioxidant, and TAC values are given as uric acid equivalents (UAE). It should be emphasized that TAC is associated mainly with low-molecular-weight antioxidants (tocopherols, glutathione, uric acid, ascorbic acid, inter alia). Enzymatic antioxidants, such as superoxide dismutase, catalase, and glutathione peroxidase do not have an impact on the values of TAC, as described in the literature [72-74].

Malondialdehyde (MDA) concentration was assayed in plasma with use of a kit (Sigma-Aldrich, cat. no. MAK085). To increase the sensitivity of the method, MDA-thiobarbituric acid adducts were extracted in $n$-butanol phase with an addition of $5 \mathrm{M} \mathrm{NaCl}$.

\subsubsection{Copper, Zinc, and Cadmium Concentration}

Copper and zinc concentration in the serum, and cadmium concentration in full blood were assayed for with use of atomic absorption spectroscopy (ASA) in (certified) Atomic Absorption Spectroscopy Laboratory of the Department and Clinic of Internal and Occupational Diseases, Wroclaw Medical University (Wroclaw, Poland). The assays were performed with use of SOLAAR M6 (Solaar House, Cambridge, UK).

\subsubsection{Genotyping}

DNA was isolated from $\mathrm{K}_{2}$ EDTA full blood, with use of Syngen Blood/Cell DNA Mini Kit (Syngen Biotech, cat. no. SY221012, Wroclaw, Poland). The isolated DNA was portioned and stored in $-80{ }^{\circ} \mathrm{C}$, in PCR test tubes (Genoplast, cat. no. GBPCR-02-NFT, Rokocin, Poland).

Genotyping of five single nucleotide polymorphisms (SNPs): rs2234694 (SOD1), rs5746105 (SOD2), rs4880 (SOD2), rs927450 (SOD2), rs8192287 (SOD3) was performed with use of PCR-RFLP method. PCR primers were designed in silico, via Primer-BLAST, based on gene sequences found in GenBank (National Center for Biotechnology Information). The following PCR mixes of Taq DNA polymerase, dNTPs and $\mathrm{MgCl}_{2}$ were used: Gold Hot Start PCR MIX (Syngen Biotech, cat. no. SY550231, Wroclaw, Poland), Silver Taq PCR MIX (Syngen Biotech, cat. no. SY550331, Wroclaw, Poland), NZYTaq II 2x Colourless Master Mix (NZYTech, cat. no. MB35701). The concentration of each primer (Table 14) in the PCR solution was $0.6 \mu \mathrm{M}$. The PCR cycle count was 38 in each product amplification. LifeEco (BIOER) thermocycler was used for both the PCR and the restriction reaction. 
Table 14. The characteristics of primers used for genotyping (method: PCR-RFLP).

\begin{tabular}{|c|c|c|c|c|}
\hline SNP (Gene) & Primers. 5'-3' Sequence (Base Pair Count) & Melting $\mathrm{T}\left({ }^{\circ} \mathrm{C}\right)$ & Annealing $\mathrm{T}\left({ }^{\circ} \mathrm{C}\right)$ & GC Content (\%) \\
\hline \multirow[t]{2}{*}{ rs2234694 (SOD1) } & $\begin{array}{c}\text { Forward: } \\
\text { CTATCCAGAAAACACGGTGGGCC(23) }\end{array}$ & 64.2 & \multirow[t]{2}{*}{55.0} & 70.6 \\
\hline & $\begin{array}{c}\text { Reverse: } \\
\text { TCTATATTCAATAAATGCTACAAAACC(27) }\end{array}$ & 55.9 & & 50.0 \\
\hline \multirow[t]{2}{*}{ rs5746105 (SOD2) } & $\begin{array}{c}\text { Forward: } \\
\text { GAGCTCGGTTGATAAAACCAGGG(23) }\end{array}$ & 62.4 & \multirow[t]{2}{*}{58.0} & 52.2 \\
\hline & $\begin{array}{c}\text { Reverse: } \\
\text { ACTCAACAAATTTCATAACCCCGA(24) }\end{array}$ & 57.6 & & 37.5 \\
\hline rs4880 (SOD2) & $\begin{array}{l}\text { Forward: GCCTGCGTAGACGGTCC(17) } \\
\text { Reverse: TCGGTGACGTTCAGGTTGTT(20) }\end{array}$ & $\begin{array}{l}60.0 \\
57.3\end{array}$ & 57.0 & $\begin{array}{l}70.6 \\
50.0\end{array}$ \\
\hline rs927450 (SOD2) & $\begin{array}{c}\text { Forward: } \\
\text { CCTGGAAACCTACATTAAGACTTTG(25) } \\
\text { Reverse: CTCTGGGGCCTACACTCTTT(20) }\end{array}$ & $\begin{array}{l}57.9 \\
58.7\end{array}$ & 57.0 & $\begin{array}{l}40.0 \\
55.0\end{array}$ \\
\hline rs8192287 (SOD3) & $\begin{array}{l}\text { Forward: TTATGAGTGCGGCTAGTGCC(20) } \\
\text { Reverse: TACTCGCCCAGTGACAACAC(20) }\end{array}$ & $\begin{array}{l}60.2 \\
60.0\end{array}$ & 57.0 & $\begin{array}{l}55.0 \\
55.0 \\
55.0\end{array}$ \\
\hline
\end{tabular}

The conditions of restriction reaction are shown in Table 15. Agarose gel electrophoresis of the restriction reaction products was run in $1 \times$ TBE buffer, $\mathrm{pH} 8.2$ (Syngen Biotech, cat. no. SY521122, Wroclaw, Poland), in 120V, $60 \mathrm{~min}$, in WHSE-014 and WMHE-600 horizontal gel electrophoresis systems (C.B.S. Scientific), with use of 5.0\% Syngen High Resolution Agarose (Syngen Biotech, cat. no. SY521017, Wroclaw, Poland)—for PCR-RFLP of rs8192287 (SOD3), or 2.0-4.0\% Syngen Daily Agarose (Syngen Biotech, cat. no. SY521011, Wroclaw, Poland)—for PCR-RFLP of rs2234694 (SOD1), rs5746105 (SOD2), rs4880 (SOD2), rs927450 (SOD2), rs8192287 (SOD3). The following DNA markers were used in electrophoresis: Ultra Low Range DNA Ladder (ThermoFisher Scientific, cat. no. 10597012, Waltham, MA, USA), GeneRuler Ultra Low Range DNA Ladder (ThermoFisher Scientific, cat. no. SM1213, Waltham, MA, USA). Syngen Blue Loading Dye (Syngen Biotech, cat. no. SY521051, Wroclaw, Poland) was used for loading the samples into the wells and Syngen Green DNA Gel Stain (Syngen Biotech, cat. no. SY521031, Wroclaw, Poland) was used for the visualization of DNA in the agarose gel. An exemplary electropherogram for each SNP is shown in Appendix B (Figures A1 and A2).

Table 15. Restriction conditions (PCR-RFLP method genotyping).

\begin{tabular}{|c|c|c|c|c|}
\hline SNP (Gene) & $\begin{array}{l}\text { Amplicon } \\
\text { Length }\end{array}$ & $\begin{array}{c}\text { Restrictase } \\
\text { Restriction Site }\end{array}$ & $\begin{array}{l}\text { Restriction } \\
\text { Conditions }\end{array}$ & Genotype Restriction Fragments \\
\hline rs2234694 (SOD1) & $278 \mathrm{bp}$ & $\begin{array}{l}\text { HhaI, Thermo Fisher } \\
\text { Scientific, cat. no. } \\
\text { ER1851 }\end{array}$ & $\begin{array}{c}37.0{ }^{\circ} \mathrm{C}, 10 \mathrm{U} \text { HhaI, } \\
1.5 \mathrm{~h}\end{array}$ & $\begin{array}{c}\text { A/A: } 278 \mathrm{bp} \\
\text { A/C: } 278 \text { bp, } 207 \mathrm{bp}, 71 \mathrm{bp} \\
\text { C/C: } 207 \mathrm{bp}, 71 \mathrm{bp}\end{array}$ \\
\hline rs5746105 (SOD2) & $259 \mathrm{bp}$ & $\begin{array}{l}\text { TasI (Tsp509I), Thermo } \\
\text { Fisher Scientific, cat. } \\
\text { no. ER1351 }\end{array}$ & $\begin{array}{l}65.0^{\circ} \mathrm{C}, 3 \mathrm{U} \text { TasI, } \\
2.5 \mathrm{~h}\end{array}$ & $\begin{array}{c}\text { C/C: } 231 \mathrm{bp}, 16 \mathrm{bp}, 12 \mathrm{bp} \\
\text { C/T: } 231 \mathrm{bp}, 110 \mathrm{bp}, 121 \mathrm{bp}, 16 \mathrm{bp}, 12 \mathrm{bp} \\
\text { T/T: } 110 \mathrm{bp}, 121 \mathrm{bp}, 16 \mathrm{bp}, 12 \mathrm{bp}\end{array}$ \\
\hline rs4880 (SOD2) & $231 \mathrm{bp}$ & $\begin{array}{l}\text { BsaWI, New England } \\
\text { Biolabs, cat. no. } \\
\text { R0567S }\end{array}$ & $\begin{array}{c}60.0^{\circ} \mathrm{C}, 3 \mathrm{U} \text { BsaWI, } \\
2.5 \mathrm{~h}\end{array}$ & $\begin{array}{c}\text { C/C: } 231 \mathrm{bp} \\
\text { C/T: } 231 \mathrm{bp}, 81 \mathrm{bp}, 150 \mathrm{bp} \\
\text { T/T: } 81 \mathrm{bp}, 150 \mathrm{bp}\end{array}$ \\
\hline rs927450 (SOD2) & $83 \mathrm{bp}$ & $\begin{array}{l}\text { BstUI, Thermo Fisher } \\
\text { Scientific, cat. no. } \\
\text { ER0921 }\end{array}$ & $\begin{array}{c}37.0^{\circ} \mathrm{C}, 3 \mathrm{U} \text { BstUI, } \\
2.5 \mathrm{~h}\end{array}$ & $\begin{array}{c}\mathrm{T} / \mathrm{T}: 81 \mathrm{bp} \\
\mathrm{T} / \mathrm{C}: 81 \mathrm{bp}, 35 \mathrm{bp}, 48 \mathrm{bp} \\
\mathrm{C} / \mathrm{C}: 35 \mathrm{bp}, 48 \mathrm{bp}\end{array}$ \\
\hline rs8192287 (SOD3) & $47 \mathrm{bp}$ & $\begin{array}{l}\text { MaeIII, Sigma-Aldrich. } \\
\text { cat. no. } 10822230001\end{array}$ & $\begin{array}{c}55.0^{\circ} \mathrm{C}, 3 \mathrm{U} \text { MaeIII, } \\
2.5 \mathrm{~h}\end{array}$ & $\begin{array}{c}\text { T/T: } 47 \text { bp } \\
\text { T/G: } 47 \text { bp, } 32 \text { bp. } 15 \text { bp } \\
\text { G/G: } 32 \text { bp, } 15 \text { bp }\end{array}$ \\
\hline
\end{tabular}

\subsection{Statistical Analyses}

The obtained data was analyzed with use of STATISTICA 13.3 package, on license provided by Wroclaw Medical University. The nominal $\alpha$ value for hypothesis testing was 0.05 . Parametric tests were preferred. Shapiro-Wilk test was used for checking for normality of distribution. Levene test was used to check for equality of variance between the compared groups. In case of normality of distribution and inequality of variances, the $t$ test with Cochran-Cox correction was used for group comparison. In case of lack of normality of distribution, the Mann-Whitney (U) test was used. 
Monotonic correlations were assessed with use of Spearman's $\varrho$. Analysis of difference in genotype frequency in context of obesity was performed with use of $\chi^{2}$ test, with additional Yates correction of the $p$-value (if any expected count was $<5.00$ ).

\section{Conclusions}

In both men and women, obesity is associated with an increase in values of total antioxidative capacity in serum. Additionally, in women, lower zinc-to-copper ratio in serum and higher cadmium concentration in full blood were observed, compared to the non-obese. Increased activity of SOD2 (Mn-SOD) in plasma was observed in obese men.

Exposition to cigarette smoke is associated with an increase in SOD2 (Mn-SOD) activity and decrease in the contribution of $\mathrm{Cu}, \mathrm{Zn}-\mathrm{SOD}$ (SOD1 and SOD3) to the total SOD activity in plasma in non-obese individuals.

Men are characterized of increased SOD1 concentration and decreased SOD3 concentration in plasma, compared to women. These differences coincide with altered values of total antioxidative capacity and concentration of metals: copper, zinc in serum, and cadmium in full blood.

Genotypic variability of SNP rs4880 (SOD2) may be associated with obesity, as the C/T genotype was observed in over $90 \%$ of obese individuals. The $\mathrm{T} / \mathrm{T}$ genotype was associated with decreased total SOD activity, $\mathrm{Cu}, \mathrm{Zn}-\mathrm{SOD}$ activity, and Mn-SOD activity in the non-obese.

Genotypic variability of SNP rs2234694 (SOD1) is associated with alterations in the concentration of SOD1 and SOD2 in the obese, and in the concentration of SOD3 in the non-obese. These findings may be due to covariance between SOD1 concentration and the concentration both SOD2 (negative) and SOD3 (positive).

Author Contributions: Conceptualization, Ł.L. and H.M.; Data curation, Ł.L.; Formal analysis, Ł.L. and H.M.; Funding acquisition, Ł.L. and H.M.; Investigation, Ł.L.; Methodology, Ł.L. and M.K.; Project administration, H.M.; Supervision, M.K. and H.M.; Validation, M.K. and H.M.; Writing-original draft, Ł.L.; Writing—review \& editing, Ł.L., M.K. and H.M. All authors have read and agreed to the published version of the manuscript.

Funding: The publication was prepared under the project financed from the funds granted by the Ministry of Science and Higher Education in the "Regional Initiative of Excellence” programme for the years 2019-2022, project number 016/RID/2018/19, the amount of funding 11998 121.30 PLN.

Conflicts of Interest: The authors declare no conflict of interest. The funders had no role in the design of the study; in the collection, analyses, or interpretation of data; in the writing of the manuscript, or in the decision to publish the results.

\section{Abbreviations}

$\begin{array}{ll}\text { BMI } & \text { body mass index } \\ \text { GAPDH } & \text { glyceraldehyde 3-phosphate dehydrogenase } \\ \text { homeostatic model assessment - insulin resistance index } \\ \text { HOMA-IR } & \text { malondialdehyde } \\ \text { MDA } & \text { reactive oxygen species } \\ \text { ROS } & \text { single nucleotide polymorphism(s) } \\ \text { SNP, SNPs } & \text { superoxide dismutase } \\ \text { SOD } & \text { cytosolic, copper-zinc superoxide dismutase } \\ \text { SOD1 } & \text { mitochondrial, manganese superoxide dismutase } \\ \text { SOD2 } & \text { extracellular, copper-zinc superoxide dismutase } \\ \text { SOD3 } & \text { superoxide dismutase isozymes } \\ \text { SODs } & \text { total antioxidative capacity } \\ \text { TAC } & \text { uncoupling protein(s) } \\ \text { UCP, UCPs } & \end{array}$

\section{Appendix A}

As mentioned before, the first four tables (Table A1, Table A2, Table A3, Table A4) are in context of SNPs: rs5746105 (SOD2) and rs927450 (SOD2). None of the differences in the first four tables in this appendix were significant, given $\alpha=0.05$. The Table A5 is supplementary to the information given in Table 12 . 
Table A1. Values of selected pro- and antioxidative parameters, and concentration of selected metals, in context of genotypic variability of rs5746105 (SOD2), in non-obese individuals.

\begin{tabular}{|c|c|c|}
\hline Parameter & $\begin{array}{c}\text { C/C or C/T Genotype }(n=27) \\
(13 \text { Women. } 14 \text { Men })\end{array}$ & $\begin{array}{l}\text { T/T Genotype }(n=23) \\
\text { (16 Women. } 7 \text { Men) }\end{array}$ \\
\hline SOD1 (ng/mL) & $\{23.43 ; 33.91 ; 42.18\}$ & $\{18.86 ; 28.40 ; 38.24\}$ \\
\hline SOD1 (ng/mg total protein) & $\{0.160 ; \mathbf{0 . 2 6 6} ; 0.354\}$ & $\{0.161 ; \mathbf{0 . 2 1 2} ; 0.324\}$ \\
\hline SOD2 $(\mathrm{ng} / \mathrm{mL})$ & $\{21.97 ; 27.55 ; 32.85\}$ & $\{22.34 ; 24.88 ; 36.75\}$ \\
\hline SOD2 (ng/mg total protein) & $\{0.168 ; 0.199 ; 0.252\}$ & $\{0.176 ; 0.219 ; 0.287\}$ \\
\hline SOD3 (ng/mL) & $\{20.50 ; 27.79 ; 36.07\}$ & $\{25.46 ; 29.21 ; 38.12\}$ \\
\hline SOD3 (ng/mg total protein) & $\{0.158 ; 0.232 ; 0.286\}$ & $\{0.184 ; 0.259 ; 0.329\}$ \\
\hline $\mathrm{SOD}(\mathrm{U} / \mathrm{L})$ & $\{1642 ; 2301 ; 2578\}$ & $\{1602 ; 2336 ; 2763\}$ \\
\hline SOD (U/g total protein) & $\{11.61 ; \mathbf{1 7 . 3 8} ; 21.08\}$ & $\{13.20 ; 19.48 ; 22.94\}$ \\
\hline SOD (U/mg SODs) & $\{20.25 ; 24.32 ; 29.48\}$ & $\{16.66 ; 23.31 ; 33.93\}$ \\
\hline $\mathrm{Cu}, \mathrm{Zn}-\mathrm{SOD}(\mathrm{U} / \mathrm{L})$ & $\{495 ; 948 ; 1264\}$ & $\{530 ; 780 ; 1252\}$ \\
\hline $\mathrm{Cu}, \mathrm{Zn}-\mathrm{SOD}$ (U/g total protein) & $\{3.90 ; 7.04 ; 9.94\}$ & $\{4.05 ; 6.77 ; 10.29\}$ \\
\hline Cu,Zn-SOD (U/mg SOD1+SOD3) & $\{8.90 ; \mathbf{1 4 . 5 0} ; 19.03\}$ & $\{10.36 ; 15.86 ; 20.02\}$ \\
\hline Cu,Zn-SOD (\% SOD activity) & $\{27.92 ; 41.26 ; 51.40\}$ & $\{25.49 ; 35.12 ; 57.02\}$ \\
\hline $\mathrm{Mn}-\mathrm{SOD}(\mathrm{U} / \mathrm{L})$ & $\{1118 ; 1213 ; 1591\}$ & $\{979 ; \mathbf{1 2 2 6} ; 1933\}$ \\
\hline Mn-SOD (U/g total protein) & $\{8.14 ; 9.97 ; 11.52\}$ & $\{8.03 ; \mathbf{1 0 . 0 8} ; 15.92\}$ \\
\hline Mn-SOD (U/mg SOD2) & $\{39.10 ; 49.55 ; 64.51\}$ & $\{27.21 ; 40.84 ; 71.02\}$ \\
\hline TAC (mM UAE) & $\{0.266 ; \mathbf{0 . 3 1 8} ; 0.359\}$ & $\{0.249 ; \mathbf{0 . 2 9 1} ; 0.335\}$ \\
\hline MDA $(\mu \mathrm{mol} / \mathrm{L})$ & $\{4.05 ; 5.21 ; 6.64\}$ & $\{3.82 ; 4.94 ; 5.75\}$ \\
\hline $\mathrm{Cu}(\mu \mathrm{g} / \mathrm{L})$ & $\{972 ; 1025 ; 1232\}$ & $\{830 ; 986 ; 1155\}$ \\
\hline $\mathrm{Zn}(\mu \mathrm{g} / \mathrm{L})$ & $\{853 ; 982 ; 1105\}$ & $\{854 ; 926 ; 1018\}$ \\
\hline $\mathrm{Zn} / \mathrm{Cu}$ & $\{0.81 ; \mathbf{0 . 9 0} ; 1.03\}$ & $\{0.84 ; 0.98 ; 1.17\}$ \\
\hline
\end{tabular}

Values shown as: \{1st quartile; median value; 3 rd quartile\}.

Table A2. Values of selected pro- and antioxidative parameters, and concentration of selected metals, in context of genotypic variability of rs5746105 (SOD2), in obese individuals.

\begin{tabular}{|c|c|c|}
\hline Parameter & $\begin{array}{c}\text { C/C or C/T Genotype }(n=26) \\
(13 \text { Women. } 13 \text { Men) }\end{array}$ & $\begin{array}{l}\text { T/T Genotype }(n=18) \\
(7 \text { Women. } 11 \text { Men })\end{array}$ \\
\hline SOD1 (ng/mL) & $\{29.64 ; 39.83 ; 49.45\}$ & $\{30.12 ; 39.47 ; 49.28\}$ \\
\hline SOD1 (ng/mg total protein) & $\{0.225 ; \mathbf{0 . 3 3 4} ; 0.437\}$ & $\{0.274 ; 0.328 ; 0.393\}$ \\
\hline SOD2 $(\mathrm{ng} / \mathrm{mL})$ & $\{18.17 ; 22.63 ; 28.03\}$ & $\{21.66 ; 25.01 ; 30.92\}$ \\
\hline SOD2 (ng/mg total protein) & $\{0.151 ; 0.192 ; 0.252\}$ & $\{0.166 ; \mathbf{0 . 2 1 1} ; 0.279\}$ \\
\hline SOD3 (ng/mL) & $\{23.57 ; 28.96 ; 32.98\}$ & $\{25.60 ; 30.25 ; 32.67\}$ \\
\hline SOD3 (ng/mg total protein) & $\{29.64 ; 39.83 ; 49.45\}$ & $\{30.12 ; 39.47 ; 49.28\}$ \\
\hline $\mathrm{SOD}(\mathrm{U} / \mathrm{L})$ & $\{2016 ; 2219 ; 2419\}$ & $\{1697 ; 2045 ; 2477\}$ \\
\hline SOD (U/g total protein) & $\{16.96 ; 18.70 ; 20.53\}$ & $\{12.64 ; 16.55 ; 20.47\}$ \\
\hline SOD (U/mg SODs) & $\{19.53 ; 22.39 ; 26.49\}$ & $\{18.03 ; 20.06 ; 25.31\}$ \\
\hline $\mathrm{Cu}, \mathrm{Zn}-\mathrm{SOD}(\mathrm{U} / \mathrm{L})$ & $\{608 ; 772 ; 1010\}$ & $\{610 ; 750 ; 852\}$ \\
\hline $\mathrm{Cu}, \mathrm{Zn}-\mathrm{SOD}$ (U/g total protein) & $\{5.12 ; 6.07 ; 7.87\}$ & $\{4.81 ; 5.98 ; 7.65\}$ \\
\hline $\mathrm{Cu}, \mathrm{Zn}-\mathrm{SOD}$ (U/mg SOD1+SOD3) & $\{7.48 ; 11.10 ; 13.99\}$ & $\{8.04 ; 10.24 ; 13.72\}$ \\
\hline Cu,Zn-SOD (\% SOD activity) & $\{28.65 ; 34.93 ; 46.37\}$ & $\{29.47 ; 36.11 ; 39.49\}$ \\
\hline $\mathrm{Mn}-\mathrm{SOD}(\mathrm{U} / \mathrm{L})$ & $\{1140 ; 1457 ; 1625\}$ & $\{1047 ; 1389 ; 1460\}$ \\
\hline Mn-SOD (U/g total protein) & $\{9.71 ; \mathbf{1 1 . 7 8} ; 13.87\}$ & $\{8.96 ; 10.63 ; 12.68\}$ \\
\hline Mn-SOD (U/mg SOD2) & $\{42.12 ; 59.26 ; 80.08\}$ & $\{36.92 ; 49.58 ; 68.37\}$ \\
\hline TAC (mM UAE) & $\{0.360 ; 0.391 ; 0.454\}$ & $\{0.324 ; 0.377 ; 0.426\}$ \\
\hline $\operatorname{MDA}(\mu \mathrm{mol} / \mathrm{L})$ & $\{6.10 ; 6.58 ; 10.35\}$ & $\{5.91 ; 6.82 ; 8.46\}$ \\
\hline $\mathrm{Cu}(\mu \mathrm{g} / \mathrm{L})$ & $\{935 ; 1056 ; 1126\}$ & $\{960 ; 1148 ; 1308\}$ \\
\hline $\mathrm{Zn}(\mu \mathrm{g} / \mathrm{L})$ & $\{894 ; 967 ; 1039\}$ & $\{878 ; 936 ; 1024\}$ \\
\hline $\mathrm{Zn} / \mathrm{Cu}$ & $\{0.79 ; 0.95 ; 1.09\}$ & $\{0.72 ; \mathbf{0 . 8 8} ; 1.01\}$ \\
\hline
\end{tabular}


Table A3. Values of selected pro- and antioxidative parameters, and concentration of selected metals, in context of genotypic variability of rs927450 (SOD2), in non-obese individuals.

\begin{tabular}{|c|c|c|c|}
\hline Parameter & $\begin{array}{l}\text { C/C Genotype }(n=12) \\
(8 \text { Women. } 4 \text { Men })\end{array}$ & $\begin{array}{l}\text { T/C Genotype }(n=22) \\
(12 \text { Women. } 10 \text { Men })\end{array}$ & $\begin{array}{l}\text { T/T Genotype }(n=16) \\
(9 \text { Women. } 7 \text { Men) }\end{array}$ \\
\hline SOD1 (ng/mL) & $\{19.32 ; 33.48 ; 37.87\}$ & $\{20.69 ; 32.43 ; 49.33\}$ & $\{17.95 ; 26.22 ; 36.78\}$ \\
\hline SOD1 (ng/mg total protein) & $\{0.178 ; 0.241 ; 0.326\}$ & $\{0.161 ; 0.243 ; 0.354\}$ & $\{0.146 ; \mathbf{0 . 1 7 7} ; 0.270\}$ \\
\hline SOD2 (ng/mL) & $\{22.29 ; 25.93 ; 29.93\}$ & $\{19.97 ; 24.31 ; 36.72\}$ & $\{22.34 ; 28.30 ; 33.44\}$ \\
\hline SOD2 (ng/mg total protein) & $\{0.186 ; 0.217 ; 0.279\}$ & $\{0.159 ; 0.215 ; 0.269\}$ & $\{0.160 ; \mathbf{0 . 2 0 3} ; 0.289\}$ \\
\hline SOD3 (ng/mL) & $\{20.64 ; 25.73 ; 33.03\}$ & $\{20.84 ; 29.98 ; 40.05\}$ & $\{18.80 ; 29.21 ; 36.24\}$ \\
\hline SOD3 (ng/mg total protein) & $\{0.165 ; 0.235 ; 0.319\}$ & $\{0.158 ; 0.250 ; 0.310\}$ & $\{0.160 ; 0.245 ; 0.270\}$ \\
\hline SOD $(\mathrm{U} / \mathrm{L})$ & $\{2106 ; 2347 ; 2590\}$ & $\{1602 ; 2337 ; 2685\}$ & $\{1539 ; \mathbf{1 8 8 2} ; 2430\}$ \\
\hline SOD (U/g total protein) & $\{15.94 ; 20.16 ; 23.07\}$ & $\{12.37 ; 19.18 ; 20.71\}$ & $\{9.97 ; 13.60 ; 20.19\}$ \\
\hline SOD (U/mg SODs) & $\{23.81 ; 27.00 ; 32.74\}$ & $\{17.25 ; 21.64 ; 33.57\}$ & $\{17.95 ; 25.27 ; 30.50\}$ \\
\hline $\mathrm{Cu}, \mathrm{Zn}-\mathrm{SOD}(\mathrm{U} / \mathrm{L})$ & $\{604 ; 1106 ; 1388\}$ & $\{530 ; 702 ; 1105\}$ & $\{436 ; 991 ; 1164\}$ \\
\hline $\mathrm{Cu}, \mathrm{Zn}-\mathrm{SOD}$ (U/g total protein) & $\{4.88 ; 9.98 ; 11.86\}$ & $\{3.98 ; 5.73 ; 9.68\}$ & $\{2.72 ; 7.49 ; 8.96\}$ \\
\hline $\begin{array}{c}\mathrm{Cu}, \mathrm{Zn}-\mathrm{SOD}(\mathrm{U} / \mathrm{mg} \\
\mathrm{SOD} 1+\mathrm{SOD} 3)\end{array}$ & $\{11.46 ; 14.58 ; 23.50\}$ & $\{7.47 ; 13.52 ; 18.25\}$ & $\{9.51 ; \mathbf{1 7 . 3 7} ; 19.11\}$ \\
\hline Cu,Zn-SOD (\% SOD activity) & $\{29.13 ; 42.88 ; 56.25\}$ & $\{25.49 ; 33.76 ; 50.56\}$ & $\{29.15 ; 37.86 ; 56.22\}$ \\
\hline $\mathrm{Mn}-\mathrm{SOD}(\mathrm{U} / \mathrm{L})$ & $\{1067 ; \mathbf{1 3 5 6} ; 1650\}$ & $\{1004 ; 1251 ; 1885\}$ & $\{1060 ; 1182 ; 1545\}$ \\
\hline Mn-SOD (U/g total protein) & $\{9.40 ; 11.07 ; 12.43\}$ & $\{8.12 ; 9.98 ; 15.76\}$ & $\{6.08 ; 9.60 ; 10.94\}$ \\
\hline Mn-SOD (U/mg SOD2) & $\{38.05 ; 48.09 ; 65.04\}$ & $\{30.27 ; 43.54 ; 71.55\}$ & $\{33.91 ; 41.48 ; 54.32\}$ \\
\hline TAC (mM UAE) & $\{0.214 ; 0.282 ; 0.321\}$ & $\{0.266 ; 0.296 ; 0.345\}$ & $\{0.265 ; \mathbf{0 . 3 3 1} ; 0.389\}$ \\
\hline $\operatorname{MDA}(\mu \mathrm{mol} / \mathrm{L})$ & $\{3.82 ; 5.06 ; 5.98\}$ & $\{3.86 ; 5.13 ; 6.18\}$ & $\{3.99 ; 5.11 ; 6.33\}$ \\
\hline $\mathrm{Cu}(\mu \mathrm{g} / \mathrm{L})$ & $\{904 ; 976 ; 1175\}$ & $\{853 ; 1010 ; 1184\}$ & $\{988 ; 1026 ; 1141\}$ \\
\hline $\mathrm{Zn}(\mu \mathrm{g} / \mathrm{L})$ & $\{821 ; 854 ; 928\}$ & $\{902 ; 988 ; 1043\}$ & $\{874 ; 981 ; 1062\}$ \\
\hline $\mathrm{Zn} / \mathrm{Cu}$ & $\{0.75 ; 0.87 ; 1.09\}$ & $\{0.84 ; 0.95 ; 1.09\}$ & $\{0.86 ; 0.91 ; 1.03\}$ \\
\hline
\end{tabular}

Values shown as: \{1st quartile; median value; 3 rd quartile\}.

Table A4. Values of selected pro- and antioxidative parameters, and concentration of selected metals, in context of genotypic variability of rs927450 (SOD2), in obese individuals.

\begin{tabular}{|c|c|c|c|}
\hline Parameter & $\begin{array}{l}\text { C/C Genotype }(n=9) \\
(4 \text { Women. } 5 \text { Men })\end{array}$ & $\begin{array}{l}\text { T/C Genotype }(n=22) \\
(10 \text { Women. } 12 \text { Men) }\end{array}$ & $\begin{array}{l}\text { T/T Genotype }(n=13) \\
(6 \text { Women. } 7 \text { Men) }\end{array}$ \\
\hline SOD1 (ng/mL) & $\{31.89 ; 43.10 ; 50.97\}$ & $\{27.15 ; 38.32 ; 49.45\}$ & $\{33.01 ; 39.83 ; 43.27\}$ \\
\hline SOD1 (ng/mg total protein) & $\{0.309 ; \mathbf{0 . 3 5 6} ; 0.426\}$ & $\{0.215 ; \mathbf{0 . 3 0 0} ; 0.437\}$ & $\{0.274 ; \mathbf{0 . 3 3 4} ; 0.402\}$ \\
\hline SOD2 (ng/mL) & $\{21.80 ; 24.82 ; 32.85\}$ & $\{18.70 ; 22.70 ; 28.03\}$ & $\{19.69 ; 23.96 ; 28.48\}$ \\
\hline SOD2 (ng/mg total protein) & $\{0.185 ; \mathbf{0 . 2 0 8} ; 0.303\}$ & $\{0.151 ; \mathbf{0 . 1 8 8} ; 0.237\}$ & $\{0.151 ; \mathbf{0 . 1 9 4} ; 0.262\}$ \\
\hline SOD3 (ng/m) & $\{28.16 ; 31.22 ; 33.16\}$ & $\{20.09 ; 27.35 ; 31.19\}$ & $\{26.76 ; 30.60 ; 34.42\}$ \\
\hline SOD3 (ng/mg total protein) & $\{0.239 ; 0.274 ; 0.291\}$ & $\{0.147 ; 0.202 ; 0.283\}$ & $\{0.226 ; 0.272 ; 0.288\}$ \\
\hline $\mathrm{SOD}(\mathrm{U} / \mathrm{L})$ & $\{1697 ; 1953 ; 2351\}$ & $\{1995 ; \mathbf{2 1 2 8} ; 2475\}$ & $\{1993 ; 2210 ; 2324\}$ \\
\hline SOD (U/g total protein) & $\{14.62 ; 16.99 ; 20.47\}$ & $\{16.55 ; 18.70 ; 21.25\}$ & $\{14.98 ; 17.31 ; 20.23\}$ \\
\hline SOD (U/mg SODs) & $\{16.34 ; 22.95 ; 24.45\}$ & $\{19.41 ; 22.09 ; 25.99\}$ & $\{18.60 ; 21.24 ; 25.05\}$ \\
\hline $\mathrm{Cu}, \mathrm{Zn}-\mathrm{SOD}(\mathrm{U} / \mathrm{L})$ & $\{656 ; 763 ; 804\}$ & $\{610 ; 713 ; 869\}$ & $\{580 ; 774 ; 1010\}$ \\
\hline $\mathrm{Cu}, \mathrm{Zn}-\mathrm{SOD}$ (U/g total protein) & $\{5.66 ; 6.54 ; 7.05\}$ & $\{5.05 ; 5.62 ; 7.61\}$ & $\{4.79 ; 6.07 ; 8.16\}$ \\
\hline $\begin{array}{l}\mathrm{Cu}, \mathrm{Zn}-\mathrm{SOD}(\mathrm{U} / \mathrm{mg} \\
\mathrm{SOD} 1+\mathrm{SOD} 3)\end{array}$ & $\{7.76 ; 10.10 ; 13.99\}$ & $\{8.07 ; \mathbf{1 0 . 8 6} ; 14.19\}$ & $\{7.09 ; 11.10 ; 13.68\}$ \\
\hline Cu,Zn-SOD (\% SOD activity) & $\{31.95 ; 34.31 ; 38.71\}$ & $\{28.85 ; 34.17 ; 48.34\}$ & $\{33.94 ; 39.49 ; 42.81\}$ \\
\hline $\mathrm{Mn}-\mathrm{SOD}(\mathrm{U} / \mathrm{L})$ & $\{1134 ; 1346 ; 1460\}$ & $\{1125 ; 1441 ; 1603\}$ & $\{1111 ; 1431 ; 1507\}$ \\
\hline Mn-SOD (U/g total protein) & $\{9.94 ; 11.24 ; 13.37\}$ & $\{9.07 ; \mathbf{1 1 . 7 0} ; 13.87\}$ & $\{9.27 ; \mathbf{1 1 . 4 4} ; 12.07\}$ \\
\hline Mn-SOD (U/mg SOD2) & $\{41.91 ; 52.03 ; 66.87\}$ & $\{40.40 ; 56.72 ; 84.90\}$ & $\{39.70 ; 61.44 ; 72.68\}$ \\
\hline TAC (mM UAE) & $\{0.312 ; \mathbf{0 . 3 9 1} ; 0.405\}$ & $\{0.331 ; 0.371 ; 0.412\}$ & $\{0.378 ; 0.454 ; 0.463\}$ \\
\hline $\operatorname{MDA}(\mu \mathrm{mol} / \mathrm{L})$ & $\{5.98 ; 6.72 ; 8.26\}$ & $\{4.63 ; 6.53 ; 8.46\}$ & $\{6.33 ; 7.65 ; 10.74\}$ \\
\hline $\mathrm{Cu}(\mu \mathrm{g} / \mathrm{L})$ & $\{900 ; 1183 ; 1290\}$ & $\{1002 ; 1075 ; 1126\}$ & $\{927 ; 995 ; 1280\}$ \\
\hline $\mathrm{Zn}(\mu \mathrm{g} / \mathrm{L})$ & $\{881 ; 952 ; 1024\}$ & $\{900 ; 999 ; 1039\}$ & $\{788 ; 932 ; 1074\}$ \\
\hline $\mathrm{Zn} / \mathrm{Cu}$ & $\{0.72 ; 0.89 ; 1.02\}$ & $\{0.79 ; 0.94 ; 1.09\}$ & $\{0.67 ; 0.92 ; 1.05\}$ \\
\hline
\end{tabular}


Table A5. Values of selected basic clinical assays: lipidogram, CRP, glucose, insulin concentration and HOMA-IR index, in context of genotypic variability of rs2234694 (SOD1) and rs4880 (SOD2).

\begin{tabular}{|c|c|c|c|}
\hline \multicolumn{4}{|c|}{ rs2234694 (SOD1) in the Control Group } \\
\hline Parameter & $\begin{array}{l}\text { A/A Genotype }(n=43) \\
(26 \text { Women, } 17 \text { Men })\end{array}$ & $\begin{array}{l}\text { A/C Genotype }(n=7) \\
\text { (3 Women, } 4 \text { Men) }\end{array}$ & $p$ \\
\hline TChol (mg/dL) & $\{175 ; 194 ; 214\}$ & $\{184 ; 194 ; 278\}$ & 0.2533 \\
\hline $\mathrm{TG}(\mathrm{mg} / \mathrm{dL})$ & $\{70 ; 87 ; 127\}$ & $\{60 ; 92 ; 152\}$ & 0.9889 \\
\hline HDL-Chol (mg/dL) & $\{48 ; 59 ; 76\}$ & $\{47 ; 59 ; 84\}$ & 0.6443 \\
\hline LDL-Chol (mg/dL) & $\{99 ; 117 ; 130\}$ & $\{98 ; 131 ; 176\}$ & 0.2909 \\
\hline $\mathrm{CRP}(\mathrm{mg} / \mathrm{L})$ & $\{0.33 ; \mathbf{0 . 6 4} ; 1.10\}$ & $\{0.19 ; 0.93 ; 1.20\}$ & 0.7220 \\
\hline Glucose (mmol/L) & $\{4.50 ; 4.78 ; 4.94\}$ & $\{4.28 ; 4.55 ; 5.28\}$ & 0.7058 \\
\hline Insulin (mU/L) & $\{4.90 ; 7.25 ; 9.00\}$ & $\{4.70 ; 5.80 ; 10.00\}$ & 0.6046 \\
\hline HOMA-IR & $\{1.02 ; 1.50 ; 1.94\}$ & $\{0.91 ; 1.09 ; 2.25\}$ & 0.6243 \\
\hline \multicolumn{4}{|c|}{ rs2234694 (SOD1) in the Obese Group } \\
\hline Parameter & $\begin{array}{l}\text { A/A Genotype }(n=36) \\
(15 \text { Women, } 21 \text { Men) }\end{array}$ & $\begin{array}{l}\text { A/C genotype }(n=8) \\
(5 \text { Men, } 3 \text { Women) }\end{array}$ & $p$ \\
\hline TChol (mg/dL) & $\{168 ; 205 ; 228\}$ & $\{166 ; 200 ; 227\}$ & 0.8540 \\
\hline $\mathrm{TG}(\mathrm{mg} / \mathrm{dL})$ & $\{84 ; 118 ; 181\}$ & $\{120 ; 141 ; 162\}$ & 0.4158 \\
\hline HDL-Chol (mg/dL) & $\{43 ; 53 ; 64\}$ & $\{42 ; 57 ; 60\}$ & 0.9413 \\
\hline LDL-Chol (mg/dL) & $\{98 ; 115 ; 155\}$ & $\{96 ; 125 ; 142\}$ & 0.8829 \\
\hline $\mathrm{CRP}(\mathrm{mg} / \mathrm{L})$ & $\{1.03 ; 1.38 ; 3.06\}$ & $\{1.11 ; 2.84 ; 4.54\}$ & 0.6082 \\
\hline Glucose (mmol/L) & $\{4.94 ; 5.20 ; 5.61\}$ & $\{4.67 ; 4.92 ; 5.28\}$ & 0.2502 \\
\hline ** Insulin (mU/L) & $\{10.00 ; 14.60 ; 19.30\}$ & $\{7.80 ; 8.40 ; 9.90\}$ & 0.0214 \\
\hline${ }^{* *}$ HOMA-IR & $\{2.25 ; 3.21 ; 4.91\}$ & $\{1.62 ; 1.75 ; 1.85\}$ & 0.0218 \\
\hline \multicolumn{4}{|c|}{ rs4880 (SOD2) in the Control Group } \\
\hline Parameter & $\begin{array}{l}\text { C/C or C/T Genotype }(n=39) \\
(24 \text { Women, } 15 \text { Men })\end{array}$ & $\begin{array}{l}\text { T/T genotype }(n=11) \\
(5 \text { Women, } 6 \text { Men) }\end{array}$ & $p$ \\
\hline TChol (mg/dL) & $\{179 ; 197 ; 215\}$ & $\{178 ; 190 ; 242\}$ & 0.8041 \\
\hline $\mathrm{TG}(\mathrm{mg} / \mathrm{dL})$ & $\{67 ; 86 ; 115\}$ & $\{75 ; 102 ; 145\}$ & 0.3249 \\
\hline HDL-Chol (mg/dL) & $\{48 ; 59 ; 76\}$ & $\{46 ; 62 ; 77\}$ & 0.6449 \\
\hline LDL-Chol (mg/dL) & $\{98 ; 117 ; 133\}$ & $\{99 ; \mathbf{1 1 8} ; 155\}$ & 0.9906 \\
\hline CRP $(\mathrm{mg} / \mathrm{L})$ & $\{0.20 ; 0.64 ; 1.05\}$ & $\{0.41 ; 0.89 ; 1.79\}$ & 0.2008 \\
\hline ** Glucose (mmol/L) & $\{4.50 ; 4.64 ; 4.89\}$ & $\{4.72 ; 4.94 ; 5.17\}$ & 0.0326 \\
\hline ** Insulin (mU/L) & $\{4.70 ; 6.60 ; 8.20\}$ & $\{6.50 ; 10.00 ; 15.20\}$ & 0.0369 \\
\hline${ }^{* *}$ HOMA-IR & $\{0.97 ; 1.35 ; 1.84\}$ & $\{1.28 ; 2.25 ; 3.56\}$ & 0.0306 \\
\hline
\end{tabular}

Values shown as: $\{1$ st quartile; median value; 3 rd quartile $\} .{ }^{* *}$ - significant difference (median values). Please note, that this table is supplementary to Table 12 .

\section{Appendix B}

In this appendix, examples of electropherograms, used in genotyping, are shown (Figures A1 and A2). 


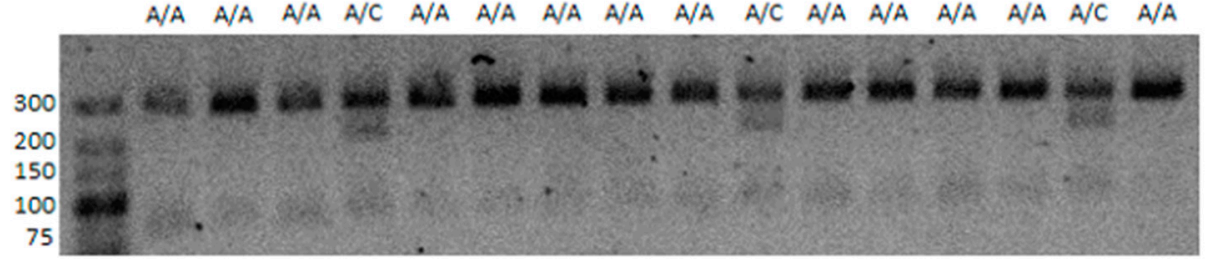

(a)

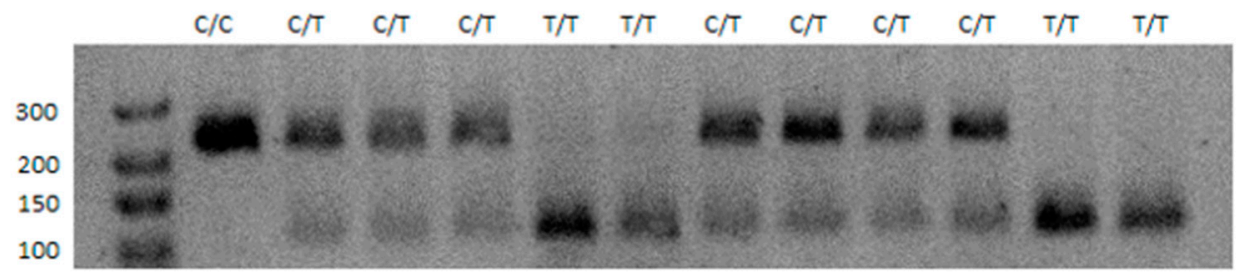

(b)

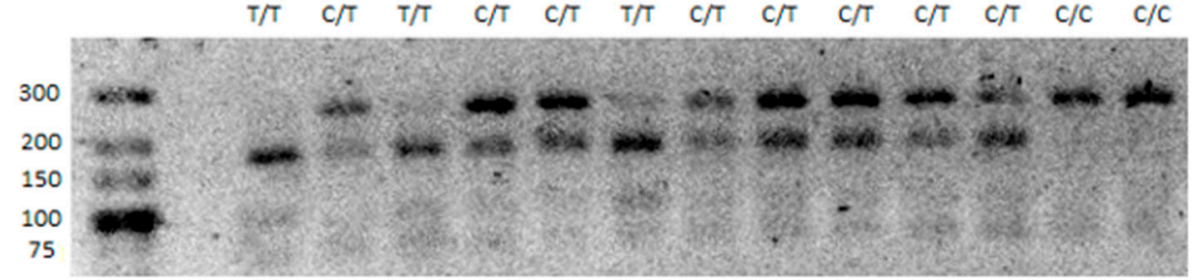

(c)

Figure A1. Examples of electropherograms used in the genotyping of: rs2234694 (SOD1) (a), rs5746105 (SOD2) (b), rs4880 (SOD2) (c).

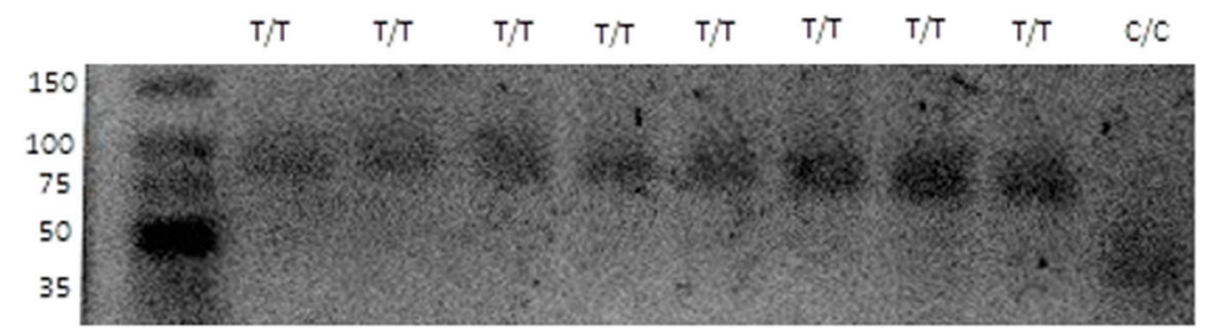

(a)

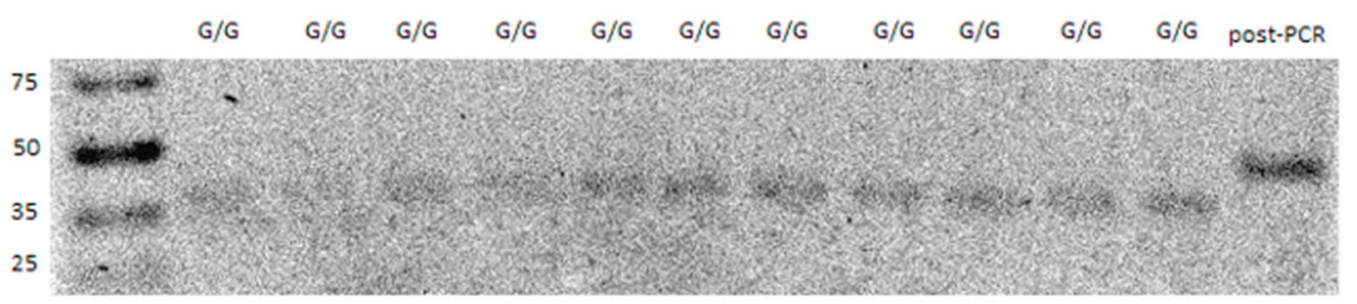

(b)

Figure A2. Examples of electropherograms used in the genotyping of: (SOD2) (a), rs8192287 (SOD3) (b).

\section{References}

1. Chen, L.; Magliano, D.J.; Zimmet, P.Z. The worldwide epidemiology of type 2 diabetes mellitus—Present and future perspectives. Nat. Rev. Endocrinol. 2012, 8, 228-236. [CrossRef] [PubMed]

2. Rabe, K.; Lehrke, M.; Parhofer, K.G.; Broedl, U.C. Adipokines and insulin resistance. Mol. Med. 2008, 14, 741-751. [CrossRef] [PubMed]

3. Fantuzzi, G. Adipose tissue, adipokines, and inflammation. J. Allergy Clin. Immunol. 2005, 115, 911-919. [CrossRef] [PubMed]

4. Kwon, H.; Pessin, J.E. Adipokines mediate inflammation and insulin resistance. Front. Endocrinol. $2013,4,71$. [CrossRef] 
5. Massiéra, F.; Bloch-Faure, M.; Ceiler, D.; Murakami, K.; Fukamizu, A.; Gasc, J.M.; Quignard-Boulange, A.; Negrel, R.; Ailhaud, G.; Seydoux, J.; et al. Adipose angiotensinogen is involved in adipose tissue growth and blood pressure regulation. FASEB J. Off. Publ. Fed. Am. Soc. Exp. Biol. 2001, 15, 2727-2729. [CrossRef]

6. Góralska, M.; Majewska-Szczepanik, M.; Szczepanik, M. Mechanizmy immunologiczne towarzyszace otyłości i ich rola w zaburzeniach metabolizmu. Postẹpy Hig. I Med. Doświadczalnej 2015, 69, 1384-1404.

7. Tangvarasittichai, S. Oxidative stress, insulin resistance, dyslipidemia and type 2 diabetes mellitus. World J. Diabetes 2015, 6, 456. [CrossRef]

8. Rains, J.L.; Jain, S.K. Oxidative stress, insulin signaling, and diabetes. Free Radic. Biol. Med. 2011, 50, 567-575. [CrossRef]

9. Meigs, J.B.; Larson, M.G.; Fox, C.S.; Keaney, J.F.; Vasan, R.S.; Benjamin, E.J. Association of oxidative stress, insulin resistance, and diabetes risk phenotypes: The Framingham Offspring Study. Diabetes Care 2007, 30 , 2529-2535. [CrossRef]

10. Rosca, M.G.; Mustata, T.G.; Kinter, M.T.; Ozdemir, A.M.; Kern, T.S.; Szweda, L.I.; Brownlee, M.; Monnier, V.M.; Weiss, M.F. Glycation of mitochondrial proteins from diabetic rat kidney is associated with excess superoxide formation. Am. J. Physiol. Ren. Physiol. 2005, 289. [CrossRef]

11. Hipkiss, A.R. Mitochondrial dysfunction, proteotoxicity, and aging: Causes or effects, and the possible impact of NAD+-controlled protein glycation. Adv. Clin. Chem. 2010, 50, 123-150. [CrossRef]

12. Rivera-Nieves, J.; Thompson, W.C.; Levine, R.L.; Moss, J. Thiols mediate superoxide-dependent NADH modification of glyceraldehyde-3-phosphate dehydrogenase. J. Biol. Chem. 1999, 274, 19525-19531. [CrossRef]

13. McClain, D.A.; Crook, E.D. Hexosamines and insulin resistance. Diabetes 1996, 45, 1003-1009. [CrossRef] [PubMed]

14. Chung, S.S.M.; Ho, E.C.M.; Lam, K.S.L.; Chung, S.K. Contribution of polyol pathway to diabetes-induced oxidative stress. J. Am. Soc. Nephrol. 2003, 14, S233-S236. [CrossRef] [PubMed]

15. Ravichandran, L.V.; Esposito, D.L.; Chen, J.; Quon, M.J. Protein kinase C- $\zeta$ phosphorylates insulin receptor substrate-1 and impairs its ability to activate phosphatidylinositol 3-kinase in response to insulin. J. Biol. Chem. 2001, 276, 3543-3549. [CrossRef] [PubMed]

16. Ishizuka, T.; Kajita, K.; Natsume, Y.; Kawai, Y.; Kanoh, Y.; Miura, A.; Ishizawa, M.; Uno, Y.; Morita, H.; Yasuda, K. Protein kinase C (PKC) $\beta$ modulates serine phosphorylation of insulin receptor substrate-1 (IRS-1)—effect of overexpression of PKC $\beta$ on insulin signal transduction. Endocr. Res. 2004, 30, 287-299. [CrossRef]

17. Chen, S.; Cohen, M.P.; Lautenslager, G.T.; Shearman, C.W.; Ziyadeh, F.N. Glycated albumin stimulates TGF- $\beta 1$ production and protein kinase $C$ activity in glomerular endothelial cells. Kidney Int. 2001, 59, 673-681. [CrossRef]

18. Garg, V.K.; Gupta, R.; Goyal, R.K. Hypozincemia in diabetes mellitus. J. Assoc. Physicians India 1994, 42, 720-721.

19. Chausmer, A.B. Zinc, insulin and diabetes. J. Am. Coll. Nutr. 1998, 17, 109-115. [CrossRef]

20. Abdel-Wahab, Y.H.A.; O'Harte, F.P.M.; Barnett, C.R.; Flatt, P.R. Characterization of insulin glycation in insulin-secreting cells maintained in tissue culture. J. Endocrinol. 1997, 152, 59-67. [CrossRef]

21. Myers, M.G.; Leibel, R.L.; Seeley, R.J.; Schwartz, M.W. Obesity and leptin resistance: Distinguishing cause from effect. Trends Endocrinol. Metab. 2010, 21, 643-651. [CrossRef]

22. Zhou, Y.; Rui, L. Leptin signaling and leptin resistance. Front. Med. 2013, 7, 207-222. [CrossRef] [PubMed]

23. Banks, W.A.; Coon, A.B.; Robinson, S.M.; Moinuddin, A.; Shultz, J.M.; Nakaoke, R.; Morley, J.E. Triglycerides Induce Leptin Resistance at the Blood-Brain Barrier. Diabetes 2004, 53, 1253-1260. [CrossRef] [PubMed]

24. Beck, B. Neuropeptides and obesity. Nutrition 2000, 16, 916-923. [CrossRef]

25. Perry, J.J.P.; Shin, D.S.; Getzoff, E.D.; Tainer, J.A. The structural biochemistry of the superoxide dismutases. Biochim. Biophys. Acta Proteins Proteom. 2010, 1804, 245-262. [CrossRef] [PubMed]

26. Lewandowski, Ł.; Kepinska, M.; Milnerowicz, H. Inhibition of copper-zinc superoxide dismutase activity by selected environmental xenobiotics. Environ. Toxicol. Pharmacol. 2018, 58, 105-113. [CrossRef]

27. Marklund, S. Superoxide dismutase in human tissues, cells and extracellular fluid: Clinical implications. In Clinical Implications, Free Radical, Aging, and Degenerative Diseases; Johnson, J.J., Walford, R., Harman, D., Miquel, J., Eds.; Alan R. Liss, Inc.: New York, NY, USA, 1986; pp. 509-526.

28. Marklund, S.L. Extracellular superoxide dismutase and other superoxide dismutase isoenzymes in tissues from nine mammalian species. Biochem. J. 1984, 222, 649-655. [CrossRef] 
29. Marklund, S.L.; Holme, E.; Hellner, L. Superoxide dismutase in extracellular fluids. Clin. Chim. Acta 1982, 126, 41-51. [CrossRef]

30. Lewandowski, Ł.; Kepinska, M.; Milnerowicz, H. The copper-zinc superoxide dismutase activity in selected diseases. Eur. J. Clin. Investig. 2019, 49, e13036. [CrossRef]

31. Stancill, J.S.; Happ, J.T.; Broniowska, K.A.; Hogg, N.; Corbett, J.A. Peroxiredoxin 1 plays a primary role in protecting pancreatic $\beta$-cells from hydrogen peroxide and peroxynitrite. Am. J. Physiol. Regul. Integr. Comp. Physiol. 2020, 318, R1004-R1013. [CrossRef]

32. Echtay, K.S.; Roussel, D.; Jekabsons, M.B.; Stuart, J.A. Superoxide activates mitochondrial uncoupling proteins. Nature 2002, 415, 96-99. [CrossRef]

33. Echtay, K.S.; Murphy, M.P.; Smith, R.A.J.; Talbot, D.A.; Brand, M.D. Superoxide activates mitochondrial uncoupling protein 2 from the matrix side. Studies using targeted antioxidants. J. Biol. Chem. 2002, 277, 47129-47135. [CrossRef] [PubMed]

34. Krauss, S.; Zhang, C.-Y.; Scorrano, L.; Dalgaard, L.T.; St-Pierre, J.; Grey, S.T.; Lowell, B.B. Superoxide-mediated activation of uncoupling protein 2 causes pancreatic $\beta$ cell dysfunction. J. Clin. Investig. 2003, 112, 1831-1842. [CrossRef] [PubMed]

35. Kubisch, H.M.; Wang, J.; Bray, T.M.; Phillips, J.P. Targeted overexpression of Cu/Zn superoxide dismutase protects pancreatic $\beta$-cells against oxidative stress. Diabetes 1997, 46, 1563-1566. [CrossRef]

36. Robbins, M.J.; Sharp, R.A.; Slonim, A.E.; Burr, I.M. Protection against streptozotocin-induced diabetes by superoxide dismutase. Diabetologia 1980, 18, 55-58. [CrossRef] [PubMed]

37. Gandy, S.E.; Buse, M.G.; Crouch, R.K. Protective role of superoxide dismutase against diabetogenic drugs. J. Clin. Investig. 1982, 70, 650-658. [CrossRef]

38. Asplund, K.; Grankvist, K.; Marklund, S.; Taljedal, I.B. Partial protection against streptozotocin-induced hyperglycaemia by superoxide dismutase linked to polyethylene glycol. Acta Endocrinol. 1984, 107, 390-394. [CrossRef] [PubMed]

39. Kaneto, H.; Fujitani, Y.; Matsuzawa, Y. Beneficial effects of antioxidants in diabetes: Possible protection of pancreatic Beta-cells against glucose toxicity. Diabetes 1999, 48, 2398-2406. [CrossRef]

40. Flekac, M.; Skrha, J.; Hilgertova, J.; Lacinova, Z.; Jarolimkova, M. Gene polymorphisms of superoxide dismutases and catalase in diabetes mellitus. BMC Med. Genet. 2008, 9, 30. [CrossRef]

41. Olsen, D.A.; Petersen, S.V.; Oury, T.D.; Valnickova, Z.; Thøgersen, I.B.; Kristensen, T.; Bowler, R.P.; Crapo, J.D.; Enghild, J.J. The intracellular proteolytic processing of extracellular superoxide dismutase (EC-SOD) is a two-step event. J. Biol. Chem. 2004, 279, 22152-22157. [CrossRef]

42. Sandström, J.; Nilsson, P.; Karlsson, K.; Marklund, S. 10-fold increase in human plasma extracellular superoxide dismutase content caused by a mutation in heparin-binding domain. J. Biol. Chem. 1994, 269, 19163-19166.

43. Yamada, H. Polymorphism of extracellular superoxide dismutase (EC-SOD) gene: Relation to the mutation responsible for high EC-SOD level in serum. Jpn. J. Hum. Genet. 1997, 42, 353-356. [CrossRef] [PubMed]

44. Dong, X.; Li, D.J.; Liu, H.; Zhao, Y.Y. SOD3 and eNOS genotypes are associated with SOD activity and NOx. Exp. Ther. Med. 2014, 8, 328-334. [CrossRef] [PubMed]

45. Sutton, A.; Khoury, H.; Prip-Buus, C.; Cepanec, C.; Pessayre, D.; Francoise, D. The Ala16Val genetic dimorphism modulates the import of human manganese superoxide dismutase into rat liver mitochondria. Pharm. Genom. 2003, 13, 145-157.

46. Becer, E.; Çırakoğlu, A. Association of the Ala16Val MnSOD gene polymorphism with plasma leptin levels and oxidative stress biomarkers in obese patients. Gene 2015, 568, 35-39. [CrossRef]

47. Montano, M.A.E.; Barrio Lera, J.P.; Gottlieb, M.G.V.; Schwanke, C.H.A.; Rocha, M.I.U.M.; Manica-Cattani, M.F.; Santos, G.F.; Cruz, I.B.M. Association between manganese superoxide dismutase (MnSOD) gene polymorphism and elderly obesity. Mol. Cell. Biochem. 2009, 328, 33-40. [CrossRef]

48. Junqueira, V.B.C.; Barros, S.B.M.; Chan, S.S.; Rodrigues, L.; Giavarotti, L.; Abud, R.L.; Deucher, G.P. Aging and oxidative stress. Mol. Asp. Med. 2004, 25, 5-16. [CrossRef]

49. Kumar Maurya, P.; Kumar, P.; Siddiqui, N.; Tripathi, P.; Rizvi, S.I. Age-associated changes in erythrocyte glutathione peroxidase activity: Correlation with total antioxidant potential. Indian J. Biochem. Biophys. 2010, 47, 319-321.

50. Rizvi, S.I.; Maurya, P.K. Alterations in antioxidant enzymes during aging in humans. Mol. Biotechnol. 2007, 37, 58-61. [CrossRef] 
51. Mendoza-Núñez, V.M.; Ruiz-Ramos, M.; Sánchez-Rodríguez, M.A.; Retana-Ugalde, R.; Muñoz-Sánchez, J.L. Aging-related oxidative stress in healthy humans. Tohoku J. Exp. Med. 2007, 213, 261-268. [CrossRef]

52. Di Massimo, C.; Scarpelli, P.; Di Lorenzo, N.; Caimi, G.; Di Orio, F.; Ciancarelli, M.G.T. Impaired plasma nitric oxide availability and extracellular superoxide dismutase activity in healthy humans with advancing age. Life Sci. 2006, 78, 1163-1167. [CrossRef]

53. Paik, H.Y.; Joung, H.; Lee, J.Y.; Lee, H.K.; King, J.C.; Keen, C.L. Serum extracellular superoxide dismutase activity as an indicator of zinc status in humans. Biol. Trace Elem. Res. 1999, 69, 45-57. [CrossRef] [PubMed]

54. Demirbag, R.; Yilmaz, R.; Erel, O. The association of total antioxidant capacity with sex hormones. Scand. Cardiovasc. J. 2005, 39, 172-176. [CrossRef]

55. Zhang, H.Q.; Li, N.; Zhang, Z.; Gao, S.; Yin, H.Y.; Guo, D.M.; Gao, X. Serum zinc, copper, and zinc/copper in healthy residents of Jinan. Biol. Trace Elem. Res. 2009, 131, 25-32. [CrossRef] [PubMed]

56. Helgeland, K.; Haider, T.; Jonsen, J. Copper and zinc in human serum in Norway: Relationship to geography, sex and age. Scand. J. Clin. Lab. Investig. 1982, 42, 35-39. [CrossRef]

57. Olsson, I.M.; Bensryd, I.; Lundh, T.; Ottosson, H.; Skerfving, S.; Oskarsson, A. Cadmium in blood and Urine-Impact of sex, age, dietary intake, iron status, and former smoking-Association of renal effects. Environ. Health Perspect. 2002, 110, 1185-1190. [CrossRef]

58. Sánchez, C.; López-Jurado, M.; Aranda, P.; Llopis, J. Plasma levels of copper, manganese and selenium in an adult population in southern Spain: Influence of age, obesity and lifestyle factors. Sci. Total Environ. 2010, 408, 1014-1020. [CrossRef] [PubMed]

59. Kim, Y.; Lee, B.K. Associations of blood lead, cadmium, and mercury with estimated glomerular filtration rate in the Korean general population: Analysis of 2008-2010 Korean National Health and Nutrition Examination Survey data. Environ. Res. 2012, 118, 124-129. [CrossRef] [PubMed]

60. Berglund, M.; Lindberg, A.L.; Rahman, M.; Yunus, M.; Grandér, M.; Lönnerdal, B.; Vahter, M. Gender and age differences in mixed metal exposure and urinary excretion. Environ. Res. 2011, 111, 1271-1279. [CrossRef]

61. Ruiz, P.; Mumtaz, M.; Osterloh, J.; Fisher, J.; Fowler, B.A. Interpreting NHANES biomonitoring data, cadmium. Toxicol. Lett. 2010, 198, 44-48. [CrossRef]

62. Ustundag, B.; Gungor, S.; Aygün, A.D.; Turgut, M.; Yilmaz, E. Oxidative status and serum leptin levels in obese prepubertal children. Cell Biochem. Funct. 2007, 25, 479-483. [CrossRef]

63. Torkanlou, K.; Bibak, B.; Abbaspour, A.; Abdi, H.; Saleh Moghaddam, M.; Tayefi, M.; Mohammadzadeh, E.; Safarian Bana, H.; Aghasizade, M.; Ferns, G.A.; et al. Reduced serum levels of zinc and superoxide dismutase in obese individuals. Ann. Nutr. Metab. 2016, 69, 232-236. [CrossRef] [PubMed]

64. Pandey, G.; Shihabudeen, M.S.; David, H.P.; Thirumurugan, E.; Thirumurugan, K. Association between hyperleptinemia and oxidative stress in obese diabetic subjects. J. Diabetes Metab. Disord. 2015, 14, 1-6. [CrossRef]

65. Stefanović, A.; Kotur-Stevuljević, J.; Spasić, S.; Bogavac-Stanojević, N.; Bujisić, N. The influence of obesity on the oxidative stress status and the concentration of leptin in type 2 diabetes mellitus patients. Diabetes Res. Clin. Pract. 2008, 79, 156-163. [CrossRef] [PubMed]

66. Ferro, F.E.D.; De Sousa Lima, V.B.; Soares, N.R.M.; De Sousa Almondes, K.G.; Pires, L.V.; Cozzolino, S.M.F.; Do Nascimento Marreiro, D. Parameters of metabolic syndrome and its relationship with zincemia and activities of superoxide dismutase and glutathione peroxidase in obese women. Biol. Trace Elem. Res. 2011, 143, 787-793. [CrossRef] [PubMed]

67. Erdeve, O.; Siklar, Z.; Kocaturk, P.A.; Dallar, Y.; Kavas, G.O. Antioxidant superoxide dismutase activity in obese children. Biol. Trace Elem. Res. 2004, 98, 219-227. [CrossRef]

68. Arsulesei, V.; Bulughiana, S.; Stoica, B.; Anisie, E. Circulating chemerin, oxidative stress, inflammation and insulin resistance in morbid obesity. Rev. Chim. 2017, 68, 1014-1018. [CrossRef]

69. Amirkhizi, F.; Siassi, F.; Djalali, M.; Shahraki, S. Impaired enzymatic antioxidant defense in erythrocytes of women with general and abdominal obesity. Obes. Res. Clin. Pract. 2014, 8, e26-e34. [CrossRef]

70. Karaouzene, N.; Merzouk, H.; Aribi, M.; Merzouk, S.A.; Yahia Berrouiguet, A.; Tessier, C.; Narce, M. Effects of the association of aging and obesity on lipids, lipoproteins and oxidative stress biomarkers: A comparison of older with young men. Nutr. Metab. Cardiovasc. Dis. 2011, 21, 792-799. [CrossRef]

71. Huang, Y.; Shih, C.; Huang, C.; Lin, C. Effects of cadmium on structure and enzymatic activity of Cu, Zn-SOD and oxidative status in neural cells. J. Cell. Biochem. 2006, 98, 577-589. [CrossRef] 
72. Bartosz, G. Non-enzymatic antioxidant capacity assays: Limitations of use in biomedicine. Free Radic. Res. 2010, 44, 711-720. [CrossRef]

73. Sies, H. Total antioxidant capacity: Appraisal of a concept. J. Nutr. 2007, 137, 1493-1495. [CrossRef] [PubMed]

74. Fraga, C.G.; Oteiza, P.I.; Galleano, M. In vitro measurements and interpretation of total antioxidant capacity. Biochim. Biophys. Acta Gen. Subj. 2014, 1840, 931-934. [CrossRef]

75. Caraballo, R.; Giovino, G.; Pechacek, T. Self-reported cigarette smoking vs. serum cotinine among U.S. adolescents. Nicotine Tob. Res. 2004, 6, 19-25. [CrossRef]

76. Wagenknecht, L.E.; Burke, G.L.; Perkins, L.L.; Haley, N.J.; Friedman, G.D. Misclassification of smoking status in the CARDIA study: A comparison of self-report with serum cotinine levels. Am. J. Public Health 1992, 82, 33-36. [CrossRef] [PubMed]

77. Kim, S. Overview of cotinine cutoff values for smoking status classification. Int. J. Environ. Res. Public Health 2016, 13, 1236. [CrossRef] [PubMed]

78. Scanu, A.M. Lipoprotein(a), friedewald formula, and NCEP guidelines. Am. J. Cardiol. 2001, 87, $608-609$. [CrossRef]

79. Okado-Matsumoto, A.; Fridovich, I. Assay of superoxide dismutase: Cautions relevant to the use of cytochrome c, a sulfonated tetrazolium, and cyanide. Anal. Biochem. 2001, 298, 337-342. [CrossRef]

80. Iqbal, J.; Whitney, P. Use of cyanide and diethyldithiocarbamate in the assay on superoxide dismutases. Free Radic. Biol. Med. 1991, 10, 69-77. [CrossRef]

81. Mejbaum-Katzenellenbogen, W.; Mochnacka, I. Metody oznaczania białek i aminokwasów. In Kurs Praktyczny z Biochemii; Państwowe Wydawnictwo Naukowe: Warszawa, Poland, 1966.

(C) 2020 by the authors. Licensee MDPI, Basel, Switzerland. This article is an open access article distributed under the terms and conditions of the Creative Commons Attribution (CC BY) license (http://creativecommons.org/licenses/by/4.0/). 